OPEN ACCESS

Edited by:

Gianluca Carnevale

University of Modena and Reggio

Emilia, Italy

Reviewed by:

Hong Ouyang,

Sun Yat-sen University, China

Marco Tatullo,

University of Bari Medical School, Italy

Kazuo Takayama,

Kyoto University, Japan

${ }^{*}$ Correspondence:

Yan-Ru Lou

yanru_lou@fudan.edu.cn

Xiaoqiang Xiang

xiangxq@fudan.edu.cn

t'These authors have contributed equally to this work and share first authorship

FThese authors have contributed equally to this work and share second authorship

\$Lead contact

Specialty section:

This article was submitted to

Stem Cell Research,

a section of the journal

Frontiers in Cell and Developmental

Biology

Received: 17 June 2021

Accepted: 10 August 2021

Published: 09 September 2021

Citation:

Bogacheva MS, Harjumäki R, Flander E, Taalas A, Bystriakova MA, Yliperttula $M$, Xiang $X$, Leung AW and

Lou Y-R (2021) Differentiation of Human Pluripotent Stem Cells Into

Definitive Endoderm Cells in Various

Flexible Three-Dimensional Cell Culture Systems: Possibilities and Limitations.

Front. Cell Dev. Biol. 9:726499. doi: 10.3389/fcell.2021.726499

\section{Differentiation of Human Pluripotent} Stem Cells Into Definitive Endoderm Cells in Various Flexible Three-Dimensional Cell Culture Systems: Possibilities and Limitations

\author{
Mariia S. Bogacheva ${ }^{1 \dagger}$, Riina Harjumäki ${ }^{1 \dagger}$, Emilia Flander ${ }^{1 \neq}$, Ara Taalas ${ }^{1 \neq}$, \\ Margarita A. Bystriakova ${ }^{1}$, Marjo Yliperttula ${ }^{1}$, Xiaoqiang Xiang ${ }^{2 *}$, Alan W. Leung ${ }^{3}$ and \\ Yan-Ru Lou ${ }^{1,2 * \$}$ \\ ${ }^{1}$ Division of Pharmaceutical Biosciences, Drug Research Program, Faculty of Pharmacy, University of Helsinki, Helsinki, \\ Finland, ${ }^{2}$ Department of Clinical Pharmacy and Drug Administration, School of Pharmacy, Fudan University, Shanghai, \\ China, ${ }^{3}$ Yale Stem Cell Center, Department of Genetics, Yale University, New Haven, CT, United States
}

The generation of human stem cell-derived spheroids and organoids represents a major step in solving numerous medical, pharmacological, and biological challenges. Due to the advantages of three-dimensional (3D) cell culture systems and the diverse applications of human pluripotent stem cell (iPSC)-derived definitive endoderm (DE), we studied the influence of spheroid size and 3D cell culture systems on spheroid morphology and the effectiveness of DE differentiation as assessed by quantitative PCR (qPCR), flow cytometry, immunofluorescence, and computational modeling. Among the tested hydrogel-based 3D systems, we found that basement membrane extract (BME) hydrogel could not retain spheroid morphology due to dominant cell-matrix interactions. On the other hand, we found that nanofibrillar cellulose (NFC) hydrogel could maintain spheroid morphology but impeded growth factor diffusion, thereby negatively affecting cell differentiation. In contrast, suspension culture provided sufficient mass transfer and was demonstrated by protein expression assays, morphological analyses, and mathematical modeling to be superior to the hydrogel-based systems. In addition, we found that spheroid size was reversely correlated with the effectiveness of DE formation. However, spheroids of insufficient sizes failed to retain 3D morphology during differentiation in all the studied culture conditions. We hereby demonstrate how the properties of a chosen biomaterial influence the differentiation process and the importance of spheroid size control for successful human iPSC differentiation. Our study provides critical parametric information for the generation of human DE-derived, tissue-specific organoids in future studies.

Keywords: definitive endoderm, human pluripotent stem cell, suspension cell culture, nanofibrillar cellulose hydrogel, computational modeling, spheroid, organoid, basement membrane extract hydrogel 


\section{INTRODUCTION}

Spheroids and organoids are three-dimensional (3D) clusters of cells. Spheroids can be made from a variety of cells including stem cells, tumor cells, and organ-specific cells. Organoids are made from stem cells or progenitors that can self-organize into organ-specific structures (Fatehullah et al., 2016; Lou and Leung, 2018). Both spheroids and organoids can produce in vivo-like structures and thus hold great potential in human development research, disease modeling, drug research, and tissue replacement via transplantation. Spheroids and organoids can be cultured and differentiated in a 3D biomaterial or in suspension without the use of a biomaterial. The selection of an appropriate biomaterial is important for successful 3D culture. Basement membrane extract (BME), such as Matrigel ${ }^{\mathrm{TM}}$, is the most widely utilized biomaterial for the formation of spheroids/organoids from many cell types (Spence et al., 2011; Chua et al., 2014; Dye et al., 2015; Hohwieler et al., 2017). BME hydrogel is an animalderived biomaterial and can interact with various types of cells via cell membrane receptors. For potential applications of spheroids/organoids in regenerative medicine, several xenofree and chemically defined hydrogels have been developed (Gjorevski et al., 2016; Nowak et al., 2017; Broguiere et al., 2018; Candiello et al., 2018). Some of these systems generate spheroid/organoid-hydrogel constructs, which may cause issues in in vivo applications, such as hydrogel biocompatibility and biodegradation. For this reason, xeno-free hydrogels that can generate biomaterial-free cell or tissue constructs have been developed. Among them, nanofibrillar cellulose (NFC) hydrogel has been shown to support the 3D spheroid formation of human embryonic stem cells (ESCs; Lou et al., 2014), induced pluripotent stem cells (iPSCs; Lou et al., 2014), liver cancer cells HepG2 (Bhattacharya et al., 2012), and HepaRG (Malinen et al., 2014). Intact spheroids formed in NFC hydrogel can be harvested after removal of the hydrogel by utilizing a cellulase enzyme (Lou et al., 2014), thereby facilitating various downstream analyses and applications (Lou et al., 2014). NFC hydrogel displayed weak interactions with human ESCs and HepG2 cells compared with natural extracellular matrix proteins including collagens and laminins (Harjumaki et al., 2019). In contrast, suspension culture is a biomaterial-free system, usually performed in a lowadhesive culture dish or a flask for scaled-up production. Like the NFC hydrogel, suspension culture can generate scaffold-free spheroids/organoids. Suspension culture has been reported to be effective in the formation of cell aggregates and organoids (Kim et al., 2016; Bergmann et al., 2018; Kumar et al., 2019; Wimmer et al., 2019).

Although spheroid/organoid technology has been rapidly developing during the past decade, several challenges remain (Lou and Leung, 2018). One of the major challenges facing organoid technology is heterogeneity and inefficient differentiation due to uncontrolled parameters such as spheroid size and mass transfer. The heterogeneity can result in variable phenotypes and inconsistent results in downstream analyses and applications. This prompted us to study factors affecting spheroid/organoid formation. We devised the current study to investigate the $3 \mathrm{D}$ differentiation of human iPSCs into definitive endoderm (DE) cells using spheroids of different sizes cultured in various 3D systems. Human iPSC differentiation toward DE represents a critical step on the way to generate cell models for the liver, gut, pancreas, lungs, trachea, and thyroid (Zorn and Wells, 2009). Great strides have been made in the development of DE differentiation methods in the previous decades (D'Amour et al., 2005; Hay et al., 2008; Wang et al., 2015; Bogacheva et al., 2018). DE cells are generally characterized by the expression of specific markers: SRY-box 17 (SOX17), Cerberus 1 (CER1), hepatocyte nuclear factor $3 \beta$ (HNF3B, also known as FOXA2), and chemokine receptor type 4 (CXCR4). In our previous study of $\mathrm{DE}$ differentiation in two-dimensional (2D) culture, we found the most efficient protocol to involve the use of activin A in a serum-free B-27-supplemented medium for 6 days (Bogacheva et al., 2018).

The current study compares three types of 3D cell culture systems: inert hydrogel-based (NFC hydrogel), cell-interacting hydrogel-based (reduced growth factor BME), and biomaterialfree (suspension) systems for their contribution to the DE differentiation of human iPSC spheroids in different sizes.

\section{MATERIALS AND METHODS}

\section{Cell Lines}

The human iPSC line iPS(IMR90)-4 was purchased from WiCell Research Institute Inc (Madison, WI, United States), and GM23720B was purchased from Coriell Institute (United States). They were cultured on Matrigel ${ }^{\mathrm{TM}}$ (BD Biosciences) with daily replenishment of the $\mathrm{mTeSR}^{\mathrm{TM}} 1$ medium (STEMCELL ${ }^{\mathrm{TM}}$ Technologies). Subculture was performed every 4 days using Versene solution 1:5,000 (Invitrogen, 15040033) for cell detachment. Cultures were maintained at $37^{\circ} \mathrm{C}$ and at $5 \%$ $\mathrm{CO}_{2}$. Mycoplasma testing was carried out regularly by the Division of Pharmaceutical Biosciences at the University of Helsinki, Finland.

\section{Formation of Spheroids and SC Differentiation to DE}

The human iPSCs cultured on Matrigel ${ }^{\mathrm{TM}}$ in the $\mathrm{mTeSR}^{\mathrm{TM}} 1$ medium were dissociated into single cells by Accutase $^{\mathrm{TM}}$ (Millipore, SCR005). Spheroids containing a different number of cells (200, 500, and 1,000 cells per spheroid) were generated in AggreWell ${ }^{\mathrm{TM}} 400$ (STEMCELL $^{\mathrm{TM}}$ Technologies, 27845 and $34411)$ in the $\mathrm{mTeSR}^{\mathrm{TM}} 1$ medium in the presence of $10 \mu \mathrm{M}$ Rhoassociated protein kinase (ROCK)-inhibitor Y-27632 (Selleck Chemicals, S1049). After $24 \mathrm{~h}$, spheroids were collected from the AggreWell ${ }^{\mathrm{TM}_{4}} 400$ and transferred in three different conditions for further culturing. Suspension culture condition was performed in a low-attachment $3.5-\mathrm{cm}$ dish (Thermo Scientific Nunc, 174913). Hydrogel culturing was performed either in $0.55 \%$ NFC hydrogel GrowDex ${ }^{\circledR}$ (UPM-Kymmene Corporation, Helsinki, Finland) in a non-adhesive 96-well plate (Corning, 3474) or in Cultrex $^{\circledast}$ Reduced Growth Factor BME (R\&D Systems, 3533-005-02) in angiogenesis 15-well slides (ibidi, Cat\# 81501, uncoated). The cell-number-to-medium-volume ratio was kept 
the same under all the conditions. The day when DE induction started was set as day 0. DE induction was performed for 6 days in the RPMI-1640 medium (Gibco, 31870-025) supplemented with $1 \times$ GlutaMAX $^{\mathrm{TM}}$ (Gibco, 35050-038), $1 \times$ B-27 (Gibco, 17504-044), $100 \mathrm{ng} / \mathrm{ml}$ activin A (PeproTech, 120-14E), and $10 \mu \mathrm{M}$ ROCK-inhibitor Y-27632. The medium was renewed daily.

\section{Live/Dead Cell Staining}

At the end of the differentiation experiment, spheroids cultured in BME were stained with a LIVE/DEAD ${ }^{\mathrm{TM}}$ Viability/Cytotoxicity Kit for mammalian cells (Thermo Fisher Scientific, L3224) according to the instruction of the manufacturer. The dye solution consisted of Calcein AM (the final concentration was $0.5 \mu \mathrm{M}$ ) and Ethidium homodimer-1 (the final concentration was $1 \mu \mathrm{M}$ ) in the RPMI-1640 medium. The cells treated with $1 \%$ Triton X100 for $5 \mathrm{~min}$ at room temperature were used as dead cell control. Spheroids were imaged within 30-60 min after staining using a confocal microscope Leica TCS SP5II HCS A with a HC PL APO $20 \times / 0.7$ CS (air) objective. Fluorescent Calcein AM (ex/em $\sim 495 \mathrm{~nm} / 215 \mathrm{~nm}$ ) produces green fluorescence in live cells. Ethidium homodimer-1 penetrates cells with damaged membranes, binds to nucleic acids, and provides red fluorescence in dead cells (ex/em $\sim 495 \mathrm{~nm} / \sim 635 \mathrm{~nm})$.

\section{Measurement of Spheroid Diameter}

The images of spheroid morphology were taken with a phase contrast microscope (Leica DM IL LED) at $5 \times$ and $10 \times$ magnifications. Spheroid diameters were then measured with the LAS EZ software (Leica Microsystems) and ImageJ (National Institutes of Health, United States).

\section{Collection of Spheroids From the NFC Hydrogel Culture}

For downstream analyses including RNA isolation, immunofluorescence staining, and flow cytometry, spheroids were collected after removing NFC hydrogel. NFC hydrogel was removed by approximately 20 -h treatment with cellulase (UPM-Kymmene Corporation, Helsinki, Finland) by following a previously described procedure (Lou et al., 2014).

\section{RNA Isolation and cDNA Conversion}

We collected RNA samples at six time points: undifferentiated stem cells cultured in 2D (2D SC), undifferentiated stem cells in spheroids (3D SC), day 1 , day 2 , day 4 , and day 6 of differentiation. Cells and spheroids were lysed using TRI-reagent (Zymo-research, R2050-1-50), and then RNA was isolated with a Direct-zol RNA MicroPrep kit (Zymo-research, R2060) according to the instruction of the manufacturer. The concentrations of RNA samples were measured with NanoDrop ${ }^{\text {TM }}$ One (Thermo Fisher Scientific). The cDNA conversion was made with a High Capacity cDNA reverse transcription kit (Applied Biosystems, 4368814) following the instructions of the manufacturer.

\section{Quantitative PCR}

The quantitative PCR (qPCR) reactions of the obtained cDNA samples were performed on a StepOnePlus Real-Time PCR System (Applied Biosystems) using either a PowerUp SYBR Green Master Mix (Applied Biosystems, A25741) or a $\operatorname{TaqMan}^{\circledR}$ Gene Expression Master Mix (Applied Biosystems, 4369016). Ribosomal protein, large, P0 ( $R P L P 0$ ) was used as a housekeeping gene. All the used primers and TaqMan Gene Expression Assay mixes are listed in Supplementary Table 1. All primers were designed by the Primer Express v2.0 software (Applied Biosystems) (Kanninen et al., 2016a) except the primers for OCT4 (Yu et al., 2007) and HNF3B (D'Amour et al., 2005), and they were synthesized by Oligomer Oy (Helsinki, Finland) or Metabion (Planegg, Germany). The relative quantification of each target gene in comparison with the housekeeping gene was made by a standard curve method based on a published mathematical model (Pfaffl, 2001). The relative gene expression was calculated with reference to the undifferentiated human iPSCs in $2 \mathrm{D}$ culture condition.

\section{Immunofluorescent Staining of 2D Cell Culture}

After the formation of SC spheroids in AggreWell ${ }^{\mathrm{TM}} 400$ for $24 \mathrm{~h}$, they were collected, dissociated into single cells using Accutase ${ }^{\mathrm{TM}}$ (Millipore, SCR005), suspended in the $\mathrm{mTeSR}^{\mathrm{TM}} 1$ medium with $10 \mu \mathrm{M}$ ROCK-inhibitor Y-27632, and seeded on laminin-521 (LN521, Biolamina) coated black 96-well $\mu$-plates (ibidi, 89626) to form a $2 \mathrm{D}$ cell monolayer. LN521 coating was prepared by incubating $10 \mu \mathrm{g} / \mathrm{ml} \mathrm{LN521} \mathrm{diluted} \mathrm{in} 1 \times$ DPBS with $\mathrm{Ca}^{+}$and $\mathrm{Mg}^{+}$either overnight at $4^{\circ} \mathrm{C}$ (slow coating) or for $2 \mathrm{~h}$ at $37^{\circ} \mathrm{C}$ (fast coating). After cells attached for $3 \mathrm{~h}$, they were fixed in $4 \%$ paraformaldehyde for $10 \mathrm{~min}$, permeabilized with $0.1 \%$ Triton X-100 for $10 \mathrm{~min}$, and thereafter blocked with $10 \%$ normal goat or donkey serum (Millipore, Burlington, MA, United States) for $1 \mathrm{~h}$. Cells were incubated with primary antibodies overnight at $4^{\circ} \mathrm{C}$ and then with secondary antibodies conjugated with Alexa Fluor 594 or Alexa Fluor 488 (Invitrogen) for $1 \mathrm{~h}$ at room temperature. Cell nuclei were stained with DAPI (Sigma-Aldrich, D8417, $12.5 \mu \mathrm{g} / \mathrm{ml}$ in MilliQ water) for $2 \mathrm{~min}$. Primary and secondary antibodies used for immunostaining in this study are listed in Supplementary Table 2.

\section{Immunofluorescent Staining of 3D Spheroids}

After 4 days of differentiation experiments, spheroids were collected from the culture dishes and fixed in $4 \%$ paraformaldehyde for $24 \mathrm{~h}$. The next day, they were treated with $100 \%$ methanol for $2 \mathrm{~min}$, then with 20\% DMSO in methanol for $2 \mathrm{~min}$, and again with methanol for $2 \mathrm{~min}$. After that, the cells were permeabilized with $1 \%$ Triton X-100 in $1 \times$ DPBS for $2 \mathrm{~min}$ and then incubated in a Penetration Buffer (0.3 M Glycine $+20 \%$ DMSO $+0.2 \%$ (wt.) Triton X-100 in $1 \times$ DPBS) for $15 \mathrm{~min}$ with shaking. Blocking was performed with a Blocking Buffer (6\% donkey serum (Southern Biotech, 0030-01) or goat serum $($ Gibco, 16210$)+10 \%$ DMSO $+0.2 \%$ (wt.) Triton X-100 in $1 \times$ 
DPBS) at $37^{\circ} \mathrm{C}$ for $15 \mathrm{~min}$ with shaking. Then the spheroids were incubated with primary antibodies diluted in an Antibody Buffer (3\% donkey serum or goat serum $+5 \%$ DMSO $+0.2 \%$ Tween $20+10 \mu \mathrm{g} / \mathrm{ml}$ Heparin in $1 \times$ DPBS) at $37^{\circ} \mathrm{C}$ for $30 \mathrm{~min}$ with shaking. Thereafter, the spheroids were washed in $1 \times$ Washing Buffer $(0.2 \%$ Tween $20+10 \mu \mathrm{g} / \mathrm{ml}$ Heparin in $1 \times$ DPBS $)$ five times for $5 \mathrm{~min}$ each at $37^{\circ} \mathrm{C}$ with shaking. Then the spheroids were incubated with secondary antibody diluted in the Antibody Buffer at $37^{\circ} \mathrm{C}$ for $30 \mathrm{~min}$ with shaking followed by washing in the same way as after the primary antibody treatment. Nuclei were stained with DAPI (Sigma-Aldrich, D8417, $12.5 \mu \mathrm{g} / \mathrm{ml}$ in MilliQ water) for $2 \mathrm{~min}$. Finally, the spheroids were treated with $100 \%$ methanol for 2 min. Visikol HISTO-M (Visikol Inc.) was added to the spheroids. Primary and secondary antibodies used for immunostaining in this study are listed in Supplementary Table 2.

\section{Imaging of Immunostaining}

Imaging was performed on a confocal microscope Leica TCS SP5II HCS A with a HC PL APO $20 \times / 0.7$ CS (air) objective. DAPI was excited with UV (diode $405 \mathrm{~nm} / 50 \mathrm{~mW}$ ), Alexa Fluor 488 with an Argon $488 \mathrm{~nm}$ laser, and Alexa Fluor 594 with a DPSS (561 nm/20 mW) laser.

\section{Flow Cytometry}

The spheroids were dissociated using Accutase ${ }^{\mathrm{TM}}$ (Millipore, SCR005) at each time point as indicated. For CXCR4 and viability measurement, single cells were incubated with either PE Mouse Anti-Human CD184 (CXCR4) IgG2a (BD Biosciences, 561733) or PE Mouse IgG2a (BD Biosciences, 555574) at the concentrations according to the instruction of the manufacturer for $40 \mathrm{~min}$ in the dark on ice. After washing with $2 \%$ FBS (Gibco, 10270-106) in $1 \times$ DPBS, the cells were treated with $0.05 \mathrm{mg} / \mathrm{ml}$ 7-AAD Viability Staining Solution (eBioscience, 006993-50) for $5 \mathrm{~min}$ in the dark on ice. Unstained cells were used to adjust FSC, SSC, and PE-Cy5. Fluorescence compensation for the CXCR4 signal in the PE channel and the 7-AAD signal in the $\mathrm{PE}-\mathrm{Cy} 5$ channel was set in an experiment using DE cells derived in $2 \mathrm{D}$ culture by following a previously described procedure (Bogacheva et al., 2018) and dead cells produced by the treatment with various concentrations of ethanol.

Analysis of the SSEA-4 surface marker was conducted at the same time points as for CXCR4. After treatment with Accutase $^{\mathrm{TM}}$, single cells were incubated with primary Mouse Anti-SSEA-4 (Developmental Studies Hybridoma Bank, MC813-70) or Mouse IgG (PeproTech, 500-M00) at $0.2 \mu \mathrm{g} / \mathrm{ml}$ for $40 \mathrm{~min}$ on ice followed by washing with $2 \%$ FBS in $1 \times$ DPBS. Thereafter, the cells were stained with F(ab')2-Goat Anti-MousePE (eBioscience, 12-4010-82) at the concentration according to the instruction of the manufacturer for $40 \mathrm{~min}$ in the dark on ice.

Flow cytometric analysis was carried out on a BD LSRII flow cytometer (YellGrn Laser, with filter PE (586/15) or PE-Cy5 $(670 / 30))$ using the BD FACSDiva software. The calculation of positive cell percentage and cell viability and the visualization of the results were performed using the FlowLogic software (Inivai Technologies). An isotype control signal was used for gating the false-positive peak caused by unspecific binding. Unstained cells' signal was used for gating living cells.

\section{Concentration Modeling}

Nanofibrillar cellulose hydrogel-based DE differentiation was performed in 96-well plates (Corning 3474). In each well, the lower phase B contained $100 \mu \mathrm{l}$ NFC hydrogel diluted in a medium and mixed with 3D cell spheroids (Supplementary Figure 1). The upper phase A contained a $100 \mu \mathrm{l}$ medium supplemented with activin A whose half-life in vivo is $5.5 \mathrm{~min}$ (fast) and $20.3 \mathrm{~min}$ (slow) (Johnson et al., 2016). Since only phase A could be renewed daily, we used a $2 \times$ medium for phase A, which contained the RPMI-1640 medium supplemented with $2 \times$ GlutaMAX $^{\mathrm{TM}}, 2 \times \mathrm{B}-27,200 \mathrm{ng} / \mathrm{ml}$ activin $\mathrm{A}$, and $20 \mu \mathrm{M}$ ROCK-inhibitor Y-27632. To model the diffusion of the key growth factor activin A from the interface between phase A and phase $\mathrm{B}$ into phase $\mathrm{B}$, we used a computational model based on the Fick's second law of diffusion (Berg, 1993), the general formula for half-life in exponential decay (Nelson, 2013), and the linear estimation of the diffusion constant from the literature (Bhattacharya et al., 2012).

Below is the derivation of the model. First is the Fick's second law of diffusion written in terms of finite difference approximations to the derivatives as (Adams and Essex, 2009):

$$
\frac{c_{j}^{n+1}-c_{j}^{n}}{\Delta t}=D \frac{c_{j+1}^{n}-2 c_{j}^{n}+c_{j-1}^{n}}{\Delta x^{2}}
$$

where $c$ is the concentration at time point $n=$ $\{0, \Delta t, 2 \Delta t, \ldots N \Delta t\}$, at position $j=\{0, \Delta x, 2 \Delta x, \ldots J \Delta x\}$, and $D$ is the diffusion constant. By defining the constant $=\frac{D \Delta t}{\Delta x^{2}}$, Equation 1 can be expressed as:

$$
c_{j}^{n+1}=S\left(c_{j+1}^{n}+c_{j-1}^{n}\right)+(1-2 S) c_{j}^{n}
$$

Denaturation of protein growth factors is considered by including the general formula for exponential degradation, with half-life $\lambda$ for time step $\Delta t$ :

$$
c_{j}^{n+1}=\left[S\left(c_{j+1}^{n}+c_{j-1}^{n}\right)+(1-2 S) c_{j}^{n}\right]\left(\frac{1}{2}\right)^{\Delta t / \lambda}
$$

By combining the Einstein relation of kinetic theory with the definition of the viscous friction coefficient (Nelson, 2013), the diffusion coefficient can be expressed in terms of the Boltzmann constant $k_{B}$, temperature $T$, time step $\Delta t$, and mass $m$ :

$$
D=\frac{k_{B} T \Delta t}{2 m}
$$

A previous study (Bhattacharya et al., 2012) had measured the diffusion constants of Dextran with different masses in NFC hydrogel. Since we can assume that the temperature of the hydrogel had been constant in each study for Dextran diffusion, we can consider $T$ as a constant in the above equation. As all the other terms are constants, we can infer that the diffusion constant $D$ and the mass of the diffusing particle $m$ have an inverse linear correlation. Derived from the publication (Bhattacharya et al., 2012) via linear regression $\left(R^{2}\right.$ 0.999), the function takes the form:

$$
D=1.76 \cdot 10^{-7} \frac{1}{m}+1.44 \cdot 10^{-5}
$$


With these definitions, the diffusion constant $D$ could be estimated for an object of mass $m$ in NFC hydrogel. Diffusion was modeled based on Equation 3, with the diffusion constant estimated as in Equation 5 by using MatLab R2014a (8.3.0.532).

\section{Statistical Analysis}

Statistical analyses were performed using the GraphPad Prism 8 software. Statistical significance was determined by one-way analysis of variance (ANOVA) followed by Sidak's multiple comparisons test or Tukey's multiple comparisons test as recommended by the software. Kruskal-Wallis test followed by Dunn's multiple comparisons test was used in case a one-way ANOVA test could not be performed because the data did not pass a normality test. Correlation analysis was performed using a Pearson's correlation test. Differences of adjusted $p<0.05\left(^{*}\right)$, adjusted $p<0.01\left(^{* *}\right)$, adjusted $p<0.001\left(^{* * *}\right)$, and adjusted $p<0.0001(* * * *)$ were considered significant.

\section{RESULTS}

\section{Initial Spheroid Size (Cell Number) Affects Spheroid Survival During DE Differentiation}

In subconfluent $2 \mathrm{D}$ cell culture where cells are well spread into monolayers, soluble factors in differentiation media are accessible to every cell. In contrast, in $3 \mathrm{D}$ cell culture, only cells in the outermost layer of spheroids/organoids are directly accessible by media components, but cells apart from the outermost layer must rely on other means such as diffusion or active transport in order to gain access to the media. To study the influence of cell layer thickness during cell differentiation, we generated spheroids using five different initial cell numbers $(50,100,200,500$, and 1,000 cells per spheroid) using AggreWell ${ }^{\mathrm{TM}} 400$ plates that contain microfabricated wells for the formation of homogenous spheroids. The initial size of each formed spheroid can be controlled by adjusting the input cell number, which requires the dissociation of stem cell colonies into single cells. This procedure results in the breakage of cell-cell interactions and cell-membrane junctions. The consequence of these events is the change in the balance between the actin-myosin anchoring force and the contraction force. The predominance of the contraction force stimulates cell death in the case of failure on re-adhesion (Chen et al., 2010). Rho-associated protein kinase (ROCK) is involved in the process of actin-myosin contraction. Treatment with the ROCK inhibitor Y-27632 improves stem cell viability in single cell status (Watanabe et al., 2007). Prior to the spheroid formation, we treated single human iPSCs iPS(IMR90)-4 cells with Y-27632 to prevent cell death. We initially chose two 3D culture conditions, suspension (without biomaterial) and NFC hydrogel. To differentiate human iPSCs into DE cells, we adopted an activin A-based protocol that was shown to be the most effective under 2D cell culture condition (Bogacheva et al., 2018). After $24 \mathrm{~h}$ in AggreWell ${ }^{\mathrm{TM}} 400$ plates, the formed spheroids were transferred either into NFC hydrogel or into suspension in a differentiation medium supplemented with Y-27632. Y-27632 was used up to day 2 of differentiation.

We obtained only a few aggregates with the initial cell number of 50 and 100 cells per spheroid in both suspension and NFC hydrogel cultures (Figure 1). Most of the survived aggregates did not acquire a round shape and were surrounded by detached cells at day 1. Subsequently, all of them dissociated within 2 days. Due to the low survival rate of spheroids with the initial cell number of 50 and 100 cells per spheroid, we excluded them from further studies.

Spheroids with the initial cell number of 200 cells survived better in suspension culture than in NFC hydrogel with spheroid yield in suspension culture being 5.5-fold more than in NFC hydrogel at day 3. Spheroids with the initial cell number of

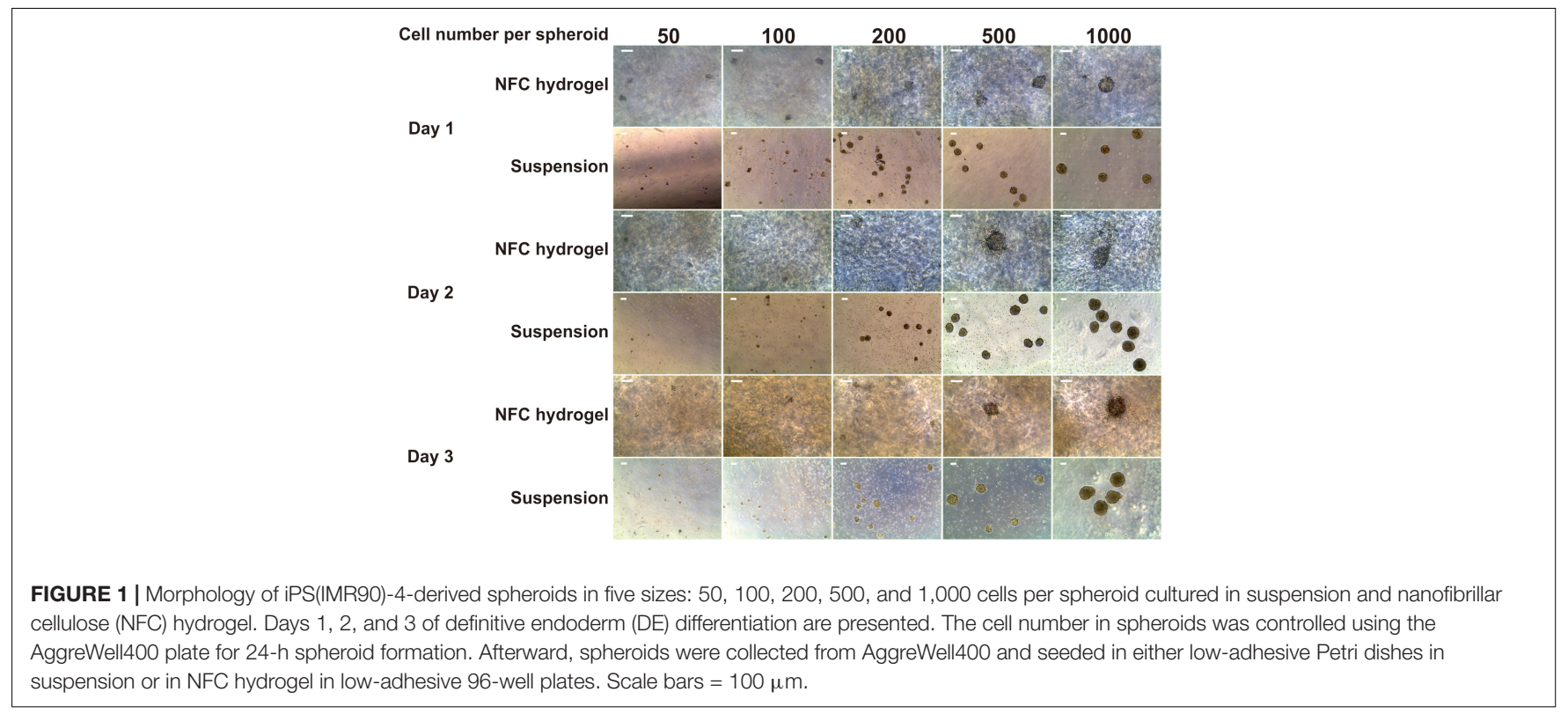


500 and 1,000 cells survived well in both conditions (Figure 1). They remained round by day 3 . The DE differentiation medium can induce cell death as observed in our previous 2D DE differentiation study (Bogacheva et al., 2018). As expected, we also noticed dead cells in the $3 \mathrm{D}$ cultures. This phenomenon was more obvious around spheroids in NFC hydrogel culture probably because dead cells were more physically restricted within the hydrogel environment.

\section{ROCK Inhibitor Y-27632 Improves Spheroid Survival During DE Differentiation}

Initially, we treated iPS(IMR90)-4-derived spheroids with Y27632 up to day 2 of differentiation but observed massive cell death (Figure 2A, single cells between spheroids) and loss of spheroid shape (Figure 2A, arrows) from day 4 onward. To obtain higher cell viability, we used Y-27632 in differentiation media for the entire period of the experiment (Figure 2B). In the presence of Y-27632, spheroids were well maintained during the 6-day differentiation. To investigate whether Y27632 may have any negative effect on DE differentiation, we studied its effect on gene expression in iPS(IMR90)-4 cells cultured under conventional 2D condition. Undifferentiated stem cells grown in colonies were dissociated with Accutase $\mathrm{TM}^{\mathrm{TM}}$ and were cultured in the $\mathrm{mTeSR}^{\mathrm{TM}} 1$ medium supplemented with Y-27632 for the first day or for 7 days (Supplementary Figure 2A). There were no statistically significant changes in the mRNA expression of all the studied genes in 1-day treated cells compared with day 0 cells (Supplementary Figure 2B). Treatment with Y-27632 for 7 days did not significantly change the gene expression of OCT4, HNF3B, CXCR4, BRACHYURY, and SOX1 (Supplementary Figure 2B). However, it significantly increased the mRNA expression of NANOG by 2.3-folds (adjusted $p=0.0327$ ) and CER1 by 4.2 -folds (adjusted $p=0.0028$ ). Earlier studies have shown the increased expression of NANOG during

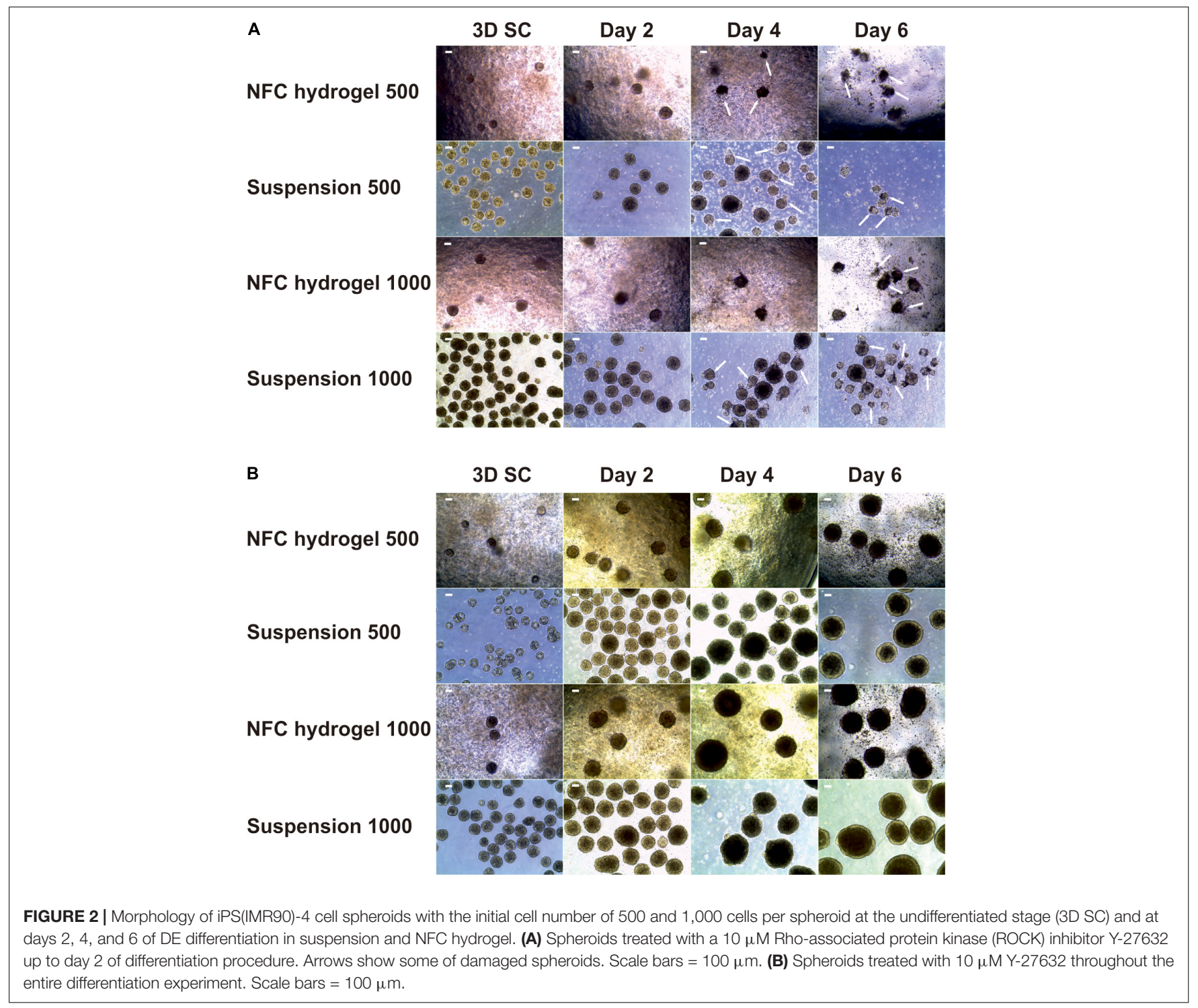


DE differentiation in 2D culture (Bogacheva et al., 2018), and NANOG was shown to be involved in early differentiation, for example, by participating in DE specification and repressing embryonic ectoderm differentiation (Teo et al., 2011; Wang et al., 2012). The upregulation of the specific DE marker CER1 is a strong indication for DE differentiation. Therefore, we chose to use Y-27632 during the entire differentiation period.

\section{D Conditions Influence Spheroid Morphology During DE Differentiation}

Based on the results from the 2D DE differentiation experiments showing that 6-day DE induction is the most effective method (Bogacheva et al., 2018), we performed 6-day DE differentiation of iPS(IMR90)-4 cells in three 3D conditions. Suspension culture in this study represents a biomaterial/scaffold-free 3D culture condition, while NFC hydrogel is an inert biomaterial that exhibits weak nonspecific interactions with cells (Harjumaki et al., 2019). Conversely, BME interacts with stem cells and DE cells directly via cell membrane receptors, so it represents an active cell-interacting biomaterial. We differentiated spheroids with the initial cell number of 500 and 1,000 cells in reduced growth factor BME and 200, 500, and 1,000 cells per spheroid in suspension or NFC hydrogel. Y-27632 was used throughout the entire experiment. At day 0, spheroids under all three culture conditions were transparent and round (Supplementary Figure 3). Hollow structures were seen in some of the spheroids at day 0 , but these structures disappeared from day 2 onward. Spheroids in BME started to disintegrate and to lose their typical spherical shape morphology from day 2 onward as a result of cell migration out of the spheroids, thereby turning part of 3D culture to resemble 2D culture (Supplementary Figures $3 \mathrm{E}, \mathbf{H}$, arrows). On the contrary, spheroids in both suspension and NFC hydrogel retained a clear spherical shape during the entire differentiation experiment (Supplementary Figures 3A-D,F,G). Some larger spheroids in suspension and NFC hydrogel became darker in the center after day 2. At day 6, we observed an increase in the spheroid size and condensed darker area in the center of spheroids grown in both NFC hydrogel and in suspension. From day 4 to day 6, cells that had migrated out of the spheroids in BME started to die, as seen by cell morphology and live/dead staining (Supplementary Figure 3). Since BME did not support the morphology of the spheroids, we excluded the BME condition from this study.

\section{Changes in Spheroid Size (Diameter) During DE Differentiation}

After excluding BME from the study, we chose suspension and NFC hydrogel conditions in the following experiments. We differentiated iPS(IMR90)-4 spheroids with the initial cell number of 200, 500, and 1,000 cells per spheroid in suspension, named 200S, 500S, and 1000S, and in NFC hydrogel, named $200 \mathrm{~N}, 500 \mathrm{~N}$, and $1000 \mathrm{~N}$, respectively. We monitored the diameters of spheroids during the 6-day differentiation (Figure 3). Spheroids under all the conditions gradually increased in size every day as measured by their diameters (Figure 3B and Supplementary Figure 4). Significant diameter increase day by day was seen more frequently in suspension culture than in NFC hydrogel culture (Supplementary Figure 4). At day 0 , undifferentiated 3D SC spheroids under the conditions 200S, 500S, and 1000S were significantly different in size (adjusted $p<0.0001$, Figure 3A), whereas in NFC hydrogel, a significant difference in size was only detected between $200 \mathrm{~N}$ and $1000 \mathrm{~N}$ (adjusted $p<0.0001$, Figure 3A). At day $1,200 \mathrm{~S}$ or $200 \mathrm{~N}$ spheroids were significantly different from $500 \mathrm{~S} / 500 \mathrm{~N}$ to $1000 \mathrm{~S} / 1000 \mathrm{~N}$ spheroids in both suspension and NFC hydrogel (Figure 3A). At days 2 and 3, we found statistically significant differences between the spheroids of all sizes in suspension (adjusted $p<0.0001$ ), but the difference in NFC hydrogel was similar to that at day 1 (Figure 3A). At day 4, we did not observe a significant difference between $500 \mathrm{~S}$ and 1000S, but we found a significant difference between $200 \mathrm{~S}$ and $500 \mathrm{~S}$, as well as between 200 S and 1000S. In NFC hydrogel, a significant difference was detected among all the conditions at day 4 (Figure 3A). Moreover, at day 4, 500S spheroids had significantly greater diameters than $500 \mathrm{~N}$ (adjusted $p=0.0452$ ). At day 5 , $200 \mathrm{~S}$ or $200 \mathrm{~N}$ spheroids were significantly different from $500 \mathrm{~S} / 500 \mathrm{~N}$ to $1000 \mathrm{~S} / 1000 \mathrm{~N}$ spheroids in both suspension and NFC hydrogel. At day 6, spheroids in suspension were all different, while in NFC hydrogel, the difference remained only between $200 \mathrm{~N}$ and $500 \mathrm{~N}$, as well as between $200 \mathrm{~N}$ and $1000 \mathrm{~N}$. In addition, $200 \mathrm{~S}$ and $500 \mathrm{~S}$ were bigger than $200 \mathrm{~N}$ and $500 \mathrm{~N}$, respectively (Figure 3A).

In summary, we found that $200 \mathrm{~S} / 200 \mathrm{~N}$ spheroids were significantly smaller than $1000 \mathrm{~S} / 1000 \mathrm{~N}$ spheroids in both suspension and NFC hydrogel during the entire differentiation experiment. However, we did not detect a significant difference between $500 \mathrm{~N}$ and $1000 \mathrm{~N}$ spheroids in NFC hydrogel at most days except at day 4. Therefore, we decided to compare $200 \mathrm{~S}$ and $200 \mathrm{~N}$ with $1000 \mathrm{~S}$ and $1000 \mathrm{~N}$ spheroids in terms of the effectiveness of DE differentiation in the subsequent immunostaining and flow cytometry experiments.

We repeated this experiment using another human iPSC line GM23720B. We differentiated GM23720B spheroids with the initial cell number of 200 and 1,000 cells per spheroid in suspension and NFC hydrogel during 4 days and assessed the dynamics of their diameter changes (Supplementary Figures 5, 6). Similar to iPS(IMR90)-4, GM23720B spheroids have gradually increased in diameters during the differentiation (Supplementary Figures 5B, 6). Also, a significant diameter increase day by day was seen more frequently in suspension culture than in NFC hydrogel culture (Supplementary Figure 6). Undifferentiated 3D SC spheroids containing 200 and 1,000 cells were significantly different in size in both suspension and NFC hydrogel (adjusted $p<0.0001$, Supplementary Figure 5A). In suspension, the difference between $200 \mathrm{~S}$ and 1000S spheroids remained until day 4 (adjusted $p<0.0001$, Supplementary Figure 5A). In NFC hydrogel, $200 \mathrm{~N}$ were significantly smaller in diameter than $1000 \mathrm{~N}$ until day 2 (adjusted $p<0.0001$, Supplementary Figure 5A). At day 4 , a significant difference between $200 \mathrm{~N}$ and $1000 \mathrm{~N}$ was detected again (adjusted $p<0.05$, Supplementary Figure 5A). At day 3 and day 4, the diameter of $1000 \mathrm{~S}$ spheroids 


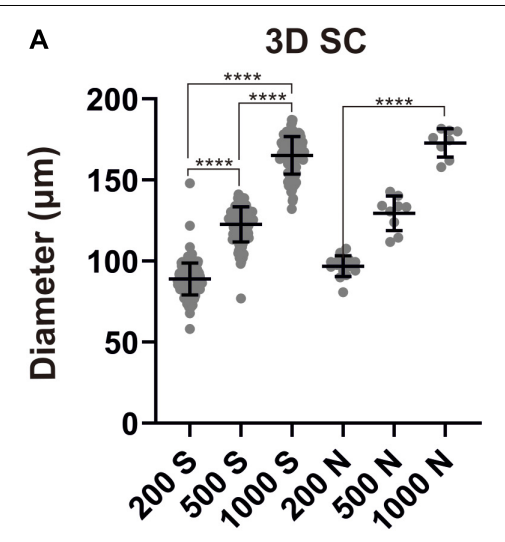

Day 3

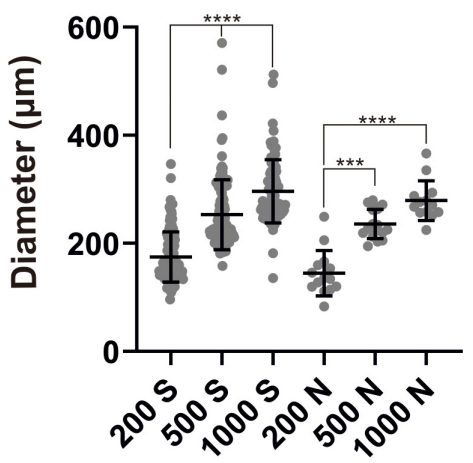

Day 6

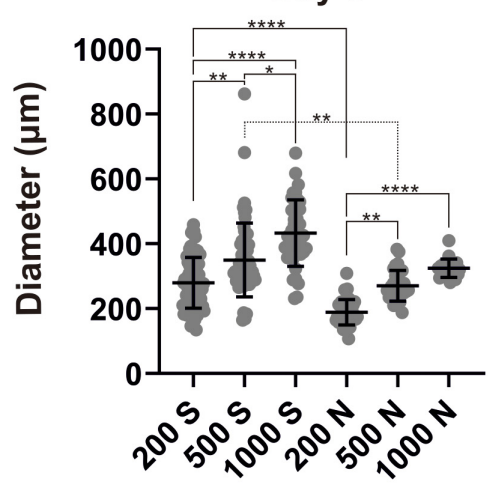

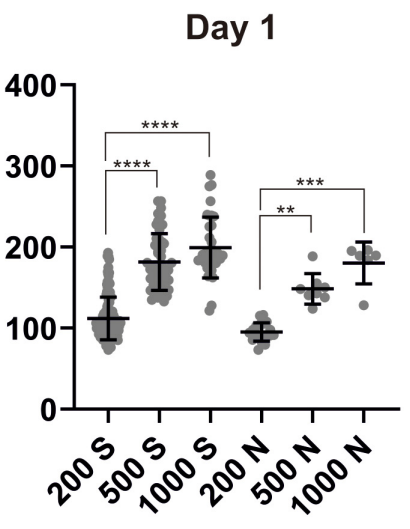

Day 4

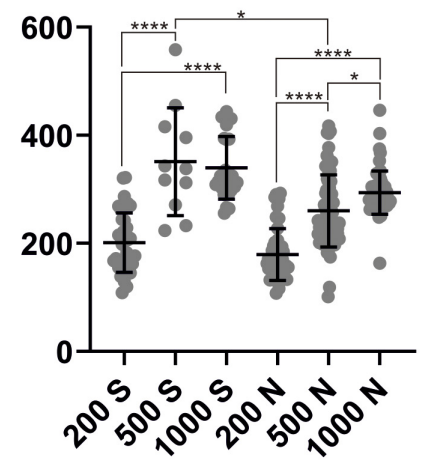

Day 2

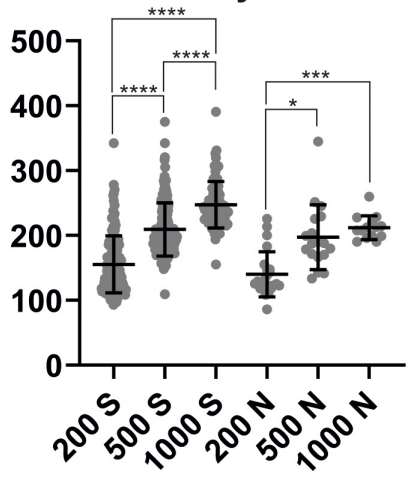

Day 5

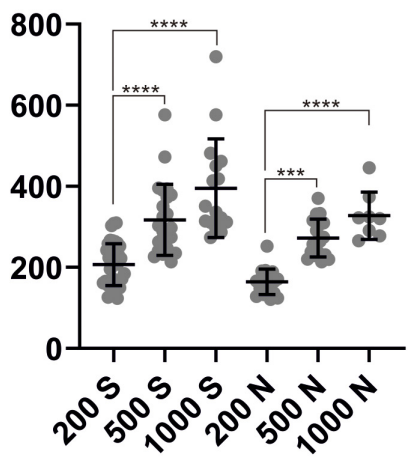

B

$\begin{array}{lccc}\begin{array}{l}\text { Condition/ } \\ \text { Timepoint }\end{array} & \text { 200S } & \text { 500S } & \text { 1000S } \\ \text { 3D SC } & 88.9 \pm 9.8 & 122.6 \pm 10.8 & 165.1 \pm 11.6 \\ \text { Day 1 } & 111.8 \pm 26.4 & 181.6 \pm 35.0 & 199.3 \pm 37.4 \\ \text { Day 2 } & 155.3 \pm 44.0 & 209.2 \pm 41.0 & 247.1 \pm 35.8 \\ \text { Day 3 } & 174.9 \pm 46.4 & 253.1 \pm 64.6 & 296.3 \pm 58.6 \\ \text { Day 4 } & 201.3 \pm 55.2 & 351.2 \pm 99.7 & 339.9 \pm 58.0 \\ \text { Day 5 } & 206.7 \pm 51.8 & 317.3 \pm 87.4 & 395.3 \pm 121.6 \\ \text { Day 6 } & 279.4 \pm 78.1 & 350.0 \pm 113.5 & 432.8 \pm 102.5\end{array}$

$200 \mathrm{~N}$
$96.8 \pm 6.4$
$95.1 \pm 11.4$
$140.1 \pm 34.7$
$144.8 \pm 42.1$
$179.0 \pm 48.0$
$164.3 \pm 31.3$
$189.0 \pm 39.0$

$500 N$

$1000 N$

$\begin{array}{ll}129.4 \pm 10.7 & 172.8 \pm 8.8 \\ 148.4 \pm 18.8 & 180.3 \pm 25.9 \\ 197.2 \pm 49.9 & 211.9 \pm 18.4 \\ 235.7 \pm 27.2 & 279.0 \pm 36.8 \\ 260.1 \pm 66.8 & 293.9 \pm 40.1 \\ 272.2 \pm 46.6 & 327.5 \pm 58.3 \\ 270.3 \pm 47.5 & 324.8 \pm 28.4\end{array}$

FIGURE 3 | Spheroid sizes during differentiation. (A) Size distribution of IPS(IMR90)-4 cell spheroids with the initial cell number of 200, 500, and 1,000 cells per spheroid during DE differentiation in suspension (S) and NFC hydrogel $(\mathrm{N})$. Diameters $(\mu \mathrm{m})$ were measured daily during the experiment. Horizontal lines are mean values, and vertical lines are SD. Because a normality test indicates that the data were not sampled from a Gaussian population, Kruskal-Wallis test followed by Dunn's multiple comparisons test was used. Statistical significance ${ }^{*}$ adjusted $p<0.05$, ${ }^{\star *}$ adjusted $p<0.01$, ${ }^{\star \star *}$ adjusted $p<0.001$, and ${ }^{* \star \star \star}$ adjusted $p<0.0001$ are shown above lines. (B) Average diameter of spheroids with the initial cell number of 200,500 , and 1,000 cells per spheroid ( $\mu \mathrm{m}) \pm S D$ at each day of the DE differentiation of iPS(IMR90)-4 cells. 
was significantly bigger than $1000 \mathrm{~N}$ (adjusted $p<0.05$, Supplementary Figure 5A).

\section{Gene Expression Profiles in iPS(IMR90)-4 Cells and Their Derivatives During DE Differentiation in 3D Conditions}

To study how spheroid size and 3D culture condition affect DE differentiation, we analyzed the gene expression profiles of mesendoderm, DE, and hepatic endoderm specific markers (Figure 4A) in 200S, 200N, 500S, 500N, 1000S, and $1000 \mathrm{~N}$ at day 0 , day 1 , day 2 , day 4 , and day 6 of the differentiation experiment. Mesendoderm is a progenitor cell stage established prior to DE specification when differentiating PSCs still retain developmental plasticity to generate either mesoderm or DE.

We first validated our approach in DE differentiation by normalizing the transcript expression levels of individual genes to their highest level during DE differentiation (Supplementary Figure 7). We confirmed that the induction of these genes followed the sequence BRACHYURY (day 1) $\rightarrow$ SOX17 (day 2) $\rightarrow H N F 3 B / C E R 1$ (day 2-4) $\rightarrow$ CXCR4 (day 4) $\rightarrow H N F 4 A$ (day 6), which was also previously observed from other DE differentiation studies (D'Amour et al., 2005; McLean et al., 2007; Teo et al., 2012).

Next, we examined the expression of individual mesendoderm and endoderm genes according to their relative expression levels to 2D SC controls, which represent undifferentiated human iPSCs, to reveal differences among conditions for different initial cell numbers and different 3D culture strategies, namely NFC hydrogel versus suspension cultures (Figures 4BG). BRACHYURY (BRA or TBXT) is a specific marker for mesendoderm lineage cells, and its expression turns off once cells become specified as DE. As expected, we observed a strong upregulation of its expression at day 1 in $200 \mathrm{~N}$ and $500 \mathrm{~N}$ cells, followed by downregulation on the next day (Figure 4B). Its expression stayed low or further reduced at day 4 and day 6 , corroborating that by day 4 , the cells were specified as DE under all the conditions.

The gene expression of the DE marker SOX17 was significantly higher in the cells at day 2 and day 4 in all the conditions in comparison with the stem cells and the cells at day 1 of differentiation (Figure 4C). At day 2, 200S and $200 \mathrm{~N}$ cells had higher SOX17 gene expression than 1000S (adjusted $p=0.034$ ) and $1000 \mathrm{~N}$ cells (adjusted $p=0.0123$ ), respectively. $500 \mathrm{~N}$ cells also showed a higher SOX17 level than $1000 \mathrm{~N}$ cells (adjusted $p=0.0271)$. At day 4, SOX17 expression significantly dropped in $200 \mathrm{~S}$ (adjusted $p=0.0128$ ), 200N (adjusted $p=0.0374$ ), $500 \mathrm{~S}$ (adjusted $p=0.0002$ ), and $500 \mathrm{~N}$ cells (adjusted $p=0.0055$ ) compared to the day 2 level. By day 6 , all the conditions did not exhibit a significant difference in SOX17 expression compared with the undifferentiated stem cells, and $1000 \mathrm{~S}$ cells even had significantly lower expression than that at day 4 (adjusted $p=0.0016)$.

The gene expression of the DE marker $H N F 3 B$ significantly increased at day 2 in all suspension conditions and $200 \mathrm{~N}$ except $500 \mathrm{~N}$ and $1000 \mathrm{~N}$, but by day 4 , it increased in all the conditions (Figure 4D). It was significantly upregulated in 1000 S cultures by day 4 compared with day 2 (adjusted $p=0.0161$ ). $1000 S$ cultures at day 4 also had higher $H N F 3 B$ expression than $500 S$ cultures (adjusted $p=0.016$ ). There was no significant increase from day 4 to day 6. The expression of $H N F 3 B$ in 200 S, 500 S, and $500 \mathrm{~N}$ cells at day 6 was not significantly different in comparison with the undifferentiated stem cells.

The gene expression of another DE marker CER1 (Iwashita et al., 2013) significantly increased in all six conditions at day 2 when compared with the stem cells and the cells at day 1 (Figure 4E). On the following days, CER1 expression did not increase significantly or even dropped in 200S cells at day 6 in comparison with day 4 (adjusted $p=0.0209$ ). $1000 \mathrm{~N}$ cells at day 6 had higher CER 1 expression than $500 \mathrm{~N}$ (adjusted $p=0.0038$ ) and 1000 S cells (adjusted $p=0.0044$ ).

CXCR4 is a well-characterized DE marker (D'Amour et al., 2005). Its expression was significantly upregulated under all the conditions at day 4 (Figure $4 \mathbf{F}$ ). By day 6 , it remained at the same level in $500 \mathrm{~N}, 1000 \mathrm{~S}$, and $1000 \mathrm{~N}$ cells and decreased in $500 \mathrm{~S}$ cells. $1000 \mathrm{~N}$ spheroids at day 4 showed higher CXCR4 expression than $200 \mathrm{~N}$ spheroids (adjusted $p=0.016$ ). 1000S spheroids at day 6 displayed higher CXCR4 expression than 200S (adjusted $p=0.0073$ ) and 500S spheroids (adjusted $p=0.0079$ ). Similarly, $1000 \mathrm{~N}$ spheroids had higher CXCR4 expression than $500 \mathrm{~N}$ cells (adjusted $p<0.0001$ ).

HNF4A isoforms are differentially expressed during development. HNF4A 1D isoform (transcribed from the P2 promoter) was earlier shown to increase in DE cells and promote DE differentiation (Hanawa et al., 2017). In the current study, we measured total HNF4A isoforms and found that their expression significantly increased at day 4 in 200S, 500S, 1000S, and $1000 \mathrm{~N}$ cells, whereas $1000 \mathrm{~S}$ cells had higher HNF4A expression than 500S cells (adjusted $p=0.0217$; Figure 4G). By day 6, HNF4A increased in $1000 \mathrm{~N}$ cells compared to the day 4 level (adjusted $p=0.0039$ ). 1000 S cells at day 6 showed higher HNF4A expression than 200S (adjusted $p=0.0179$ ) and 500S cells (adjusted $p=0.0004$ ). $1000 \mathrm{~N}$ cells had higher HNF4A expression than $500 \mathrm{~N}$ cells (adjusted $p=0.0003$ ).

Taken together, we found that the studied DE markers were significantly increased at day 2 or day 4 in comparison with the undifferentiated stem cells. Only HNF4A expression was further increased from day 4 to day 6 . Intriguingly, the expression of the DE markers SOX17, CER1, and CXCR4 started to decrease at day 6 suggesting an accelerated differentiation program compared with 2D adherent differentiation (Bogacheva et al., 2018). Hence, we selected the 4-day differentiation protocol in the following experiments. Since the PCR method determines gene expression in bulk cell populations, we next used flow cytometry and immunofluorescence staining to assess DE differentiation in individual cells.

\section{Spheroid Size and 3D Culture Conditions Affect the Efficiency and the Effectiveness of DE Formation}

Before DE differentiation, we stained pluripotency markers in iPS(IMR90)-4 spheroids after their formation in AggreWell ${ }^{\mathrm{TM}} 400$ plates. All the spheroids with 50, 100, 

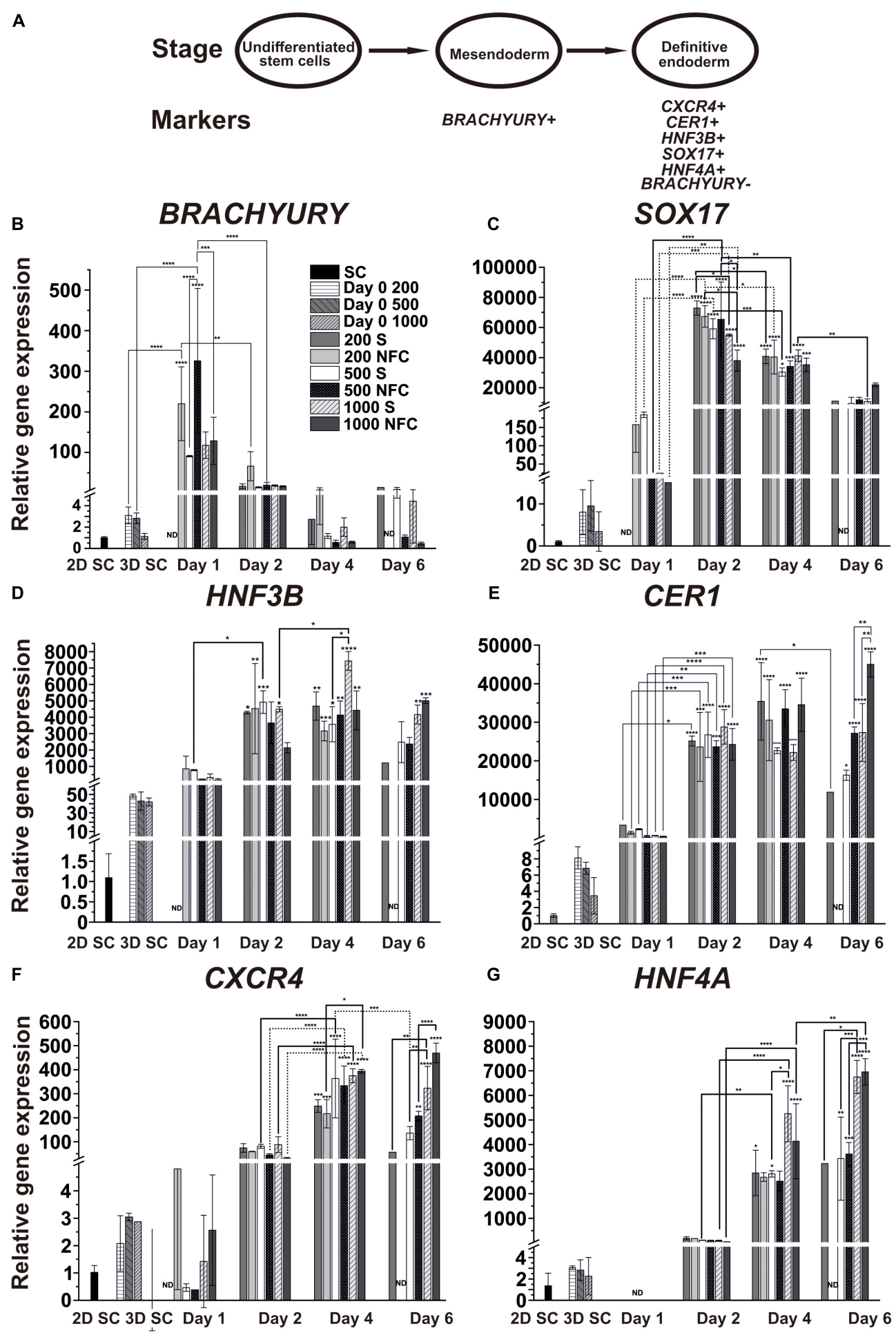

FIGURE 4 | (A) Schematic of the characteristic markers at the studied differentiation stages. (B-G) The mRNA expression patterns of the mesendoderm (BRACHYURY), DE (SOX17, HNF3B, CER1, and CXCR4), and hepatic endoderm (HNF4A) specific markers during DE differentiation of iPS(IMR90)-4 cells in 3D spheroids with the initial cell number of 200,500 , and 1000 cells per spheroid in suspension (S) and NFC hydrogel (N). Relative gene expression was measured by qPCR and normalized with RPLPO housekeeping gene. Fold inductions were calculated with the reference to the stem cell samples (2D SC). $N=2$ or 3 biological repeats. ND, no data. Error bars are SD. One-way ANOVA followed by Tukey's multiple comparisons test was used to compare between any pairs. Statistical significance *adjusted $P<0.05$, ${ }^{* *}$ adjusted $P<0.01$, ${ }^{* *}$ adjusted $P<0.001$, and ${ }^{* \star \star *}$ adjusted $P<0.0001$ in comparison with $2 \mathrm{D}$ SC are shown above bars. Statistically significant differences ${ }^{*}$ adjusted $P<0.05$, ${ }^{* *}$ adjusted $P<0.01$, ${ }^{\star * \star}$ adjusted $P<0.001$, and ${ }^{* \star * *}$ adjusted $P<0.0001$ between days 1 and 2 , days 2 and 4 , and days 4, and 6 are shown above lines. Breaks in $Y$-axis in the figure panels are BRACHYURY: 5-7, SOX17: 16-26 and 200-9000, HNF3B: 2-5 and 60-150, CER1: 10-50, CXCR4: 5-30, and HNF4A: 5-30. 
200, 500, and 1,000 cells per spheroid were positive for OCT4 and NANOG proteins (Supplementary Figure 8).

We evaluated the efficiency and the effectiveness of the DE formation by image-based analysis of SOX17, HNF3B, and HNF4A protein expression and quantitative analysis of CXCR4 expression. We conducted immunofluorescence staining for the DE markers SOX17, HNF3B, and HNF4A and the pluripotency marker OCT4 in day 4 spheroids (Figure 5 and

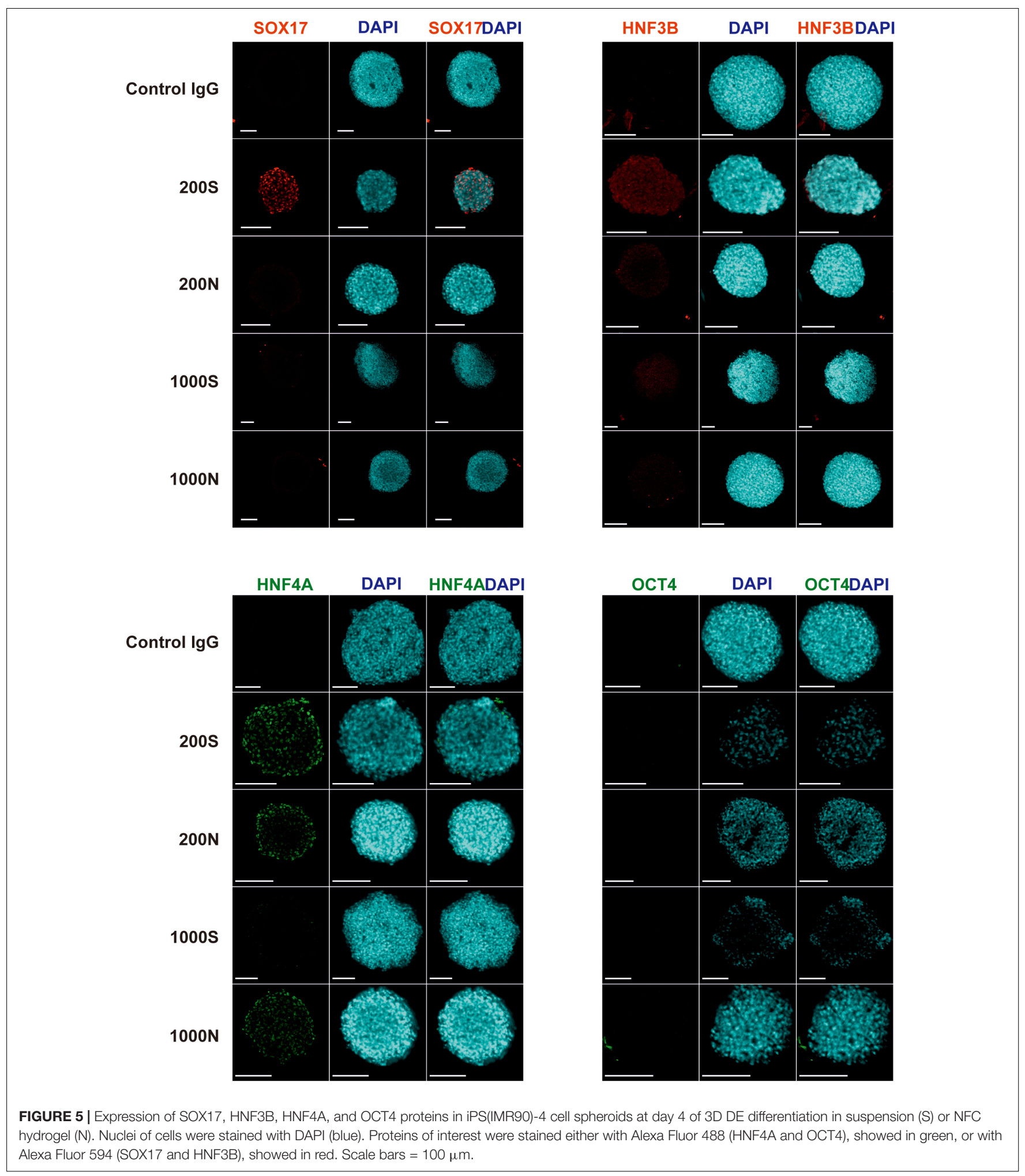


Supplementary Figure 9). 200S iPS(IMR90)-4 spheroids were positive for SOX17, HNF3B, and HNF4A (Figure 5). 200N spheroids were strongly positive for HNF4A protein and weakly so for HNF3B in the cells of the outer layers (Figure 5), which displayed similar intensity to what was observed in $2 \mathrm{D} D E$ differentiation previously (Bogacheva et al., 2018), but 200N spheroids were negative for SOX17 (Figure 5). 1000S spheroids had the weakly positive expression of HNF3B and HNF4A proteins and did not express SOX17 (Figure 5). 1000N spheroids showed strong HNF4A expression and were negative for SOX17 and HNF3B (Figure 5). None of the conditions showed OCT4 protein expression (Figure 5).

Similar to iPS(IMR90)-4 DE spheroids, OCT4 protein expression was not observed in day 4 GM23720B cell spheroids in all the conditions (Supplementary Figure 9). 200S spheroids were positive for SOX17 and HNF3B proteins while negative for HNF4A (Supplementary Figure 9). 200N and 1000S spheroids had positive signals for SOX17 (Supplementary Figure 9). 1000N spheroids demonstrated low or no expression for the stained protein markers (Supplementary Figure 9).

To quantify the differentiation in individual cells, we performed flow cytometry analysis of iPS(IMR90)-4 and GM23720B spheroids and their derivatives differentiated in suspension or NFC hydrogel at day 1, day 2, and day 4 of DE differentiation (Supplementary Figure 10). We examined the pluripotency marker SSEA4 and the DE marker CXCR4 in iPS(IMR90)-4 spheroids (Figures 6, 7, and Supplementary Figure 11) and the DE marker CXCR4 in GM23720B spheroids (Supplementary Figure 12).

The percentage of SSEA4 ${ }^{+}$cells in iPS(IMR90)-4 cell spheroids before differentiation was more than 99\% (Figures 6A, 7A), and it significantly dropped by day 4 only in spheroids cultured in suspension (adjusted $p<0.0001$ for $200 \mathrm{~S}$ and adjusted $p=0.0003$ for 1000 S, Figure 7A). We also observed that the fluorescent intensity peaks in all histograms shift to the left during the differentiation (Figure 6A and Supplementary Figure 11). At day 4,1000 S spheroids had significantly less SSEA $4^{+}$cells than $1000 \mathrm{~N}$ spheroids (adjusted $p=0.0139$, Figure 7A).

Spheroids with the initial cell number of 200 and 1,000 cells per spheroid at the stem cell stage were negative for CXCR4 protein (Figures 6B, 7B, and Supplemetary Figure 12A). The percentage of CXCR4 ${ }^{+}$iPS(IMR90)- 4 cells did not change at day 1 but rose at day 2 and then reached its maximum by day 4 (Figures 6B, 7B). Similarly, the percentage of CXCR4 ${ }^{+}$ GM23720B cells also increased at day 2 and day 4 (Supplemetary Figure 12A). At day 2, $200 \mathrm{~S}$ iPS(IMR90)-4 spheroids displayed the highest percentage of CXCR4 ${ }^{+}$cells $(67.7 \pm 8.6 \%$, Figure 7B). This percentage was significantly higher than that for $200 \mathrm{~N}$ $(49.5 \pm 4.9 \%$, adjusted $P=0.0056)$ and 1000 S spheroids (25.9 $\pm 8.6 \%$, adjusted $P<0.0001$, Figure 7B). Similarly, 200S GM23720B spheroids also displayed higher percentage of $\mathrm{CXCR}^{+}$cells $(62.7 \pm 5.8 \%)$ than that for $200 \mathrm{~N}(54.0 \pm 5.0 \%)$ and 1000 S spheroids $(55.8 \pm 5.5 \%$, Supplemetary Figure 12A). For the iPS(IMR90)-4 spheroids in NFC hydrogel, a similar trend to suspension cultures was observed: $200 \mathrm{~N}$ spheroids ( $49.5 \pm 4.9 \%)$ displayed a significantly higher percentage of CXCR4 $4^{+}$cells than $1000 \mathrm{~N}$ spheroids ( $15.2 \pm 12.4 \%$, adjusted $p<0.0001$, Figure 7B).
Furthermore, we found a negative correlation between iPS(IMR90)-4 spheroids' size and percentage of CXCR4 ${ }^{+}$cells in both NFC hydrogel ( $p=0.0225$, Figure 7C) and suspension $(p=0.0024$, Figure 7D) at day 2 of the differentiation. For GM23720B spheroids, the correlation was found between spheroids' size and percentage of CXCR4 $4^{+}$cells in suspension at day 4 of the differentiation ( $p=0.0251$, Supplementary Figure 12C).

When measuring the CXCR4 expression by flow cytometry, we also stained the cells with 7-AAD fluorescent dye to assess cell death in spheroids. We did not detect a significant difference in cell death between time points or culture conditions (Supplementary Figure 13). The percentage of dead cells in spheroids was averaged $4.6 \% \pm 1.6 \%$ under all the conditions. This indicates that poor differentiation performance of spheroids under certain culture conditions was not caused by decreased cell viability.

In summary, these data demonstrate that smaller hPSC spheroids differentiate to DE cells more efficiently than bigger spheroids, and suspension culture promotes more effective DE differentiation than NFC hydrogel.

\section{Computational Simulation of Activin A Diffusion}

Activin A used in the differentiation media is crucial for DE formation. Earlier studies showed that its effect is concentrationdependent, with high concentrations $(100 \mathrm{ng} / \mathrm{ml})$ specifying DE (D'Amour et al., 2005) and low concentrations specifying mesoderm (Schuldiner et al., 2000). The poor efficiency and effectiveness of $\mathrm{DE}$ formation from the bigger spheroids cultured in NFC hydrogel could indicate a poor diffusion of activin A due to mass transfer limitation caused either by cell masses in spheroids or by biomaterials. Simulating the diffusion of proteins within a spheroid is technically difficult to perform; thus, our model is focused on simulating the diffusion of activin A within the NFC hydrogel layer. By establishing a computational model based on Fick's second law of diffusion and an NFC hydrogelspecific linear regression model based on the Einstein relation of kinetic theory and the viscous friction coefficient, we found that only the top $0.25 \mathrm{~mm}(8 \%)$ of the NFC hydrogel layer received $100 \mathrm{ng} / \mathrm{ml}$ activin A (Figure 8). Since protein halflives in cell culture and in tissues are different (Rahman and Sadygov, 2017), our simulation using the in vivo half-life of activin A might overestimate or underestimate the activin A diffusion in vitro. Nonetheless, the simulation result indicates that activin A encountered considerable resistance to its transfer across NFC hydrogel, presumably due to hydrogel's high viscosity (Bhattacharya et al., 2012). The limited mass transfer may explain the low effectiveness of the DE differentiation in NFC hydrogel (Figure 9).

\section{DISCUSSION}

To overcome the major challenges in spheroid and organoid technology and increase the effectiveness of stem cell differentiation in $3 \mathrm{D}$ environments, we examined how $3 \mathrm{D}$ cell 


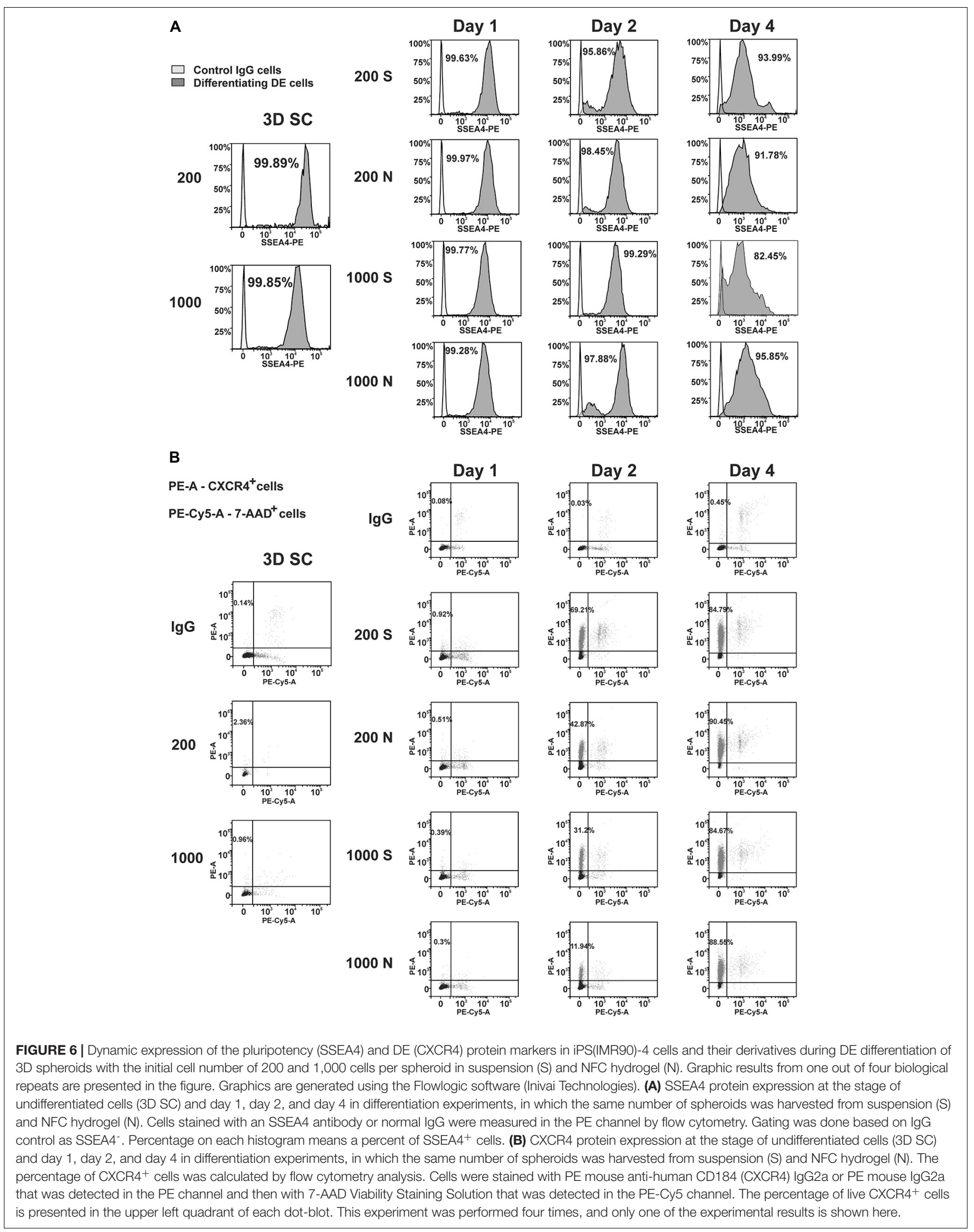



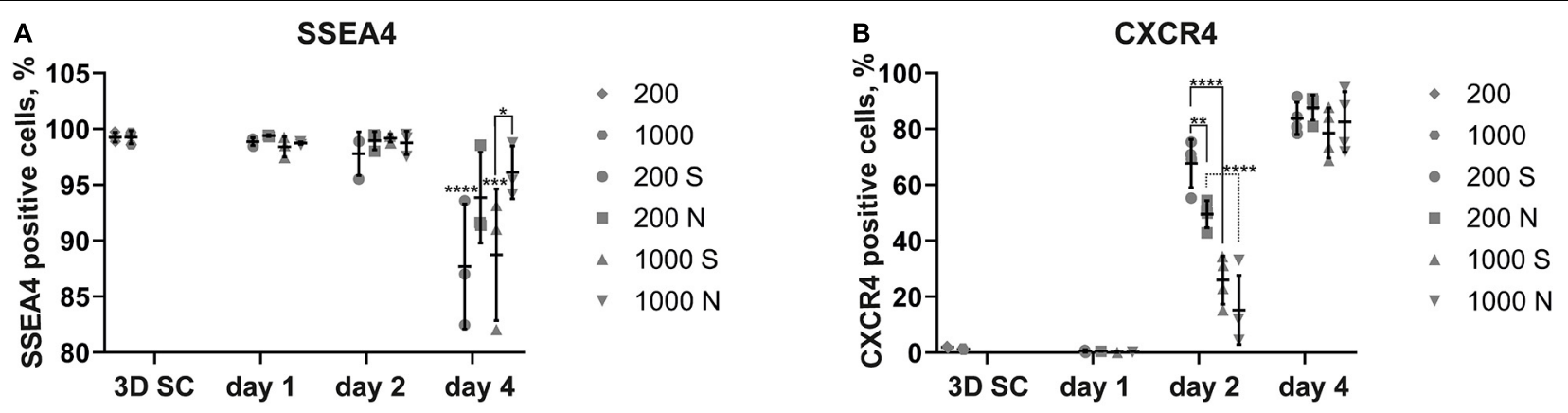

\section{c NFC hydrogel culture}
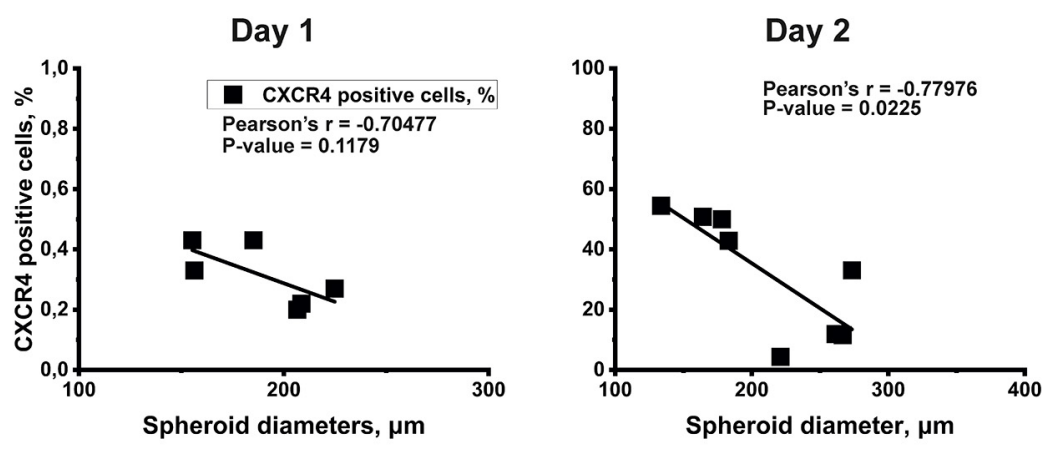

Day 4

\section{Suspension culture}
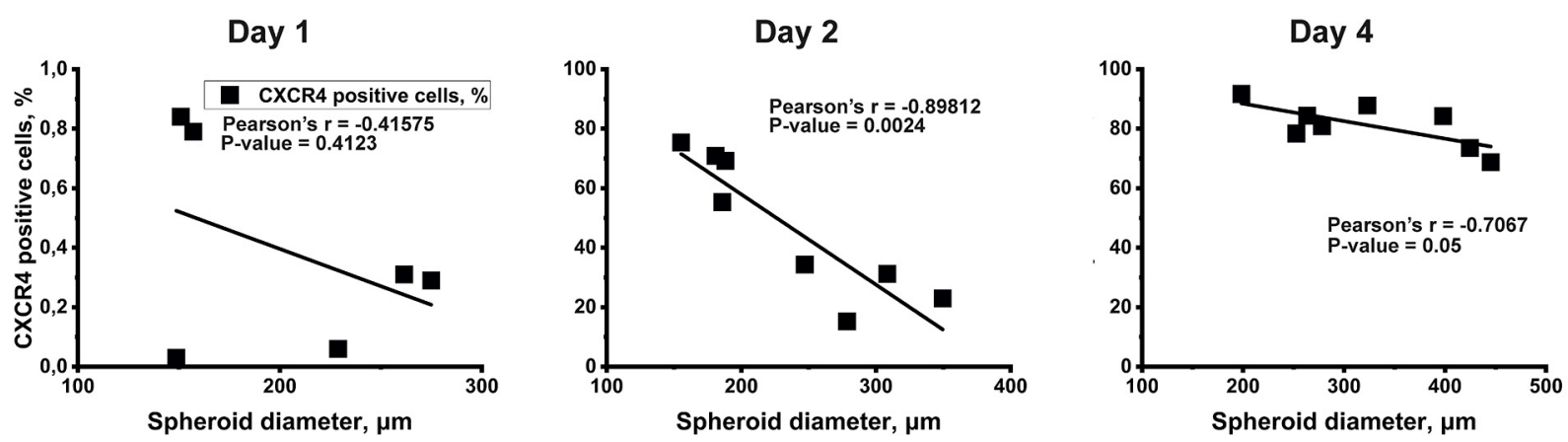

FIGURE 7 | The quantitative expression of markers and the correlation between spheroid diameters and the effectiveness of the DE differentiation in iPS(IMR90)-4 cells. (A) SSEA4 protein expression pattern in iPS(IMR90)-4 cell spheroids (3D SC) and their derivatives during 3D DE differentiation in suspension (S) and NFC hydrogel (N). $N=3$ biological repeats. Data were analyzed by one-way ANOVA followed by Sidak's multiple comparisons test. Statistical significance ${ }^{\star \star \star}$ adjusted $p<0.001$ and ${ }^{* \star *}$ adjusted $p<0.0001$ in comparison with 3D SC are shown above scatters. Statistically significant difference ${ }^{*}$ adjusted $p<0.05$ between conditions $1000 \mathrm{~S}$ and $1000 \mathrm{~N}$ at day 4 is shown above the line. (B) CXCR4 protein expression pattern in iPS(IMR90)-4 cell spheroids (3D SC) and their derivatives during 3D DE differentiation in suspension (S) and NFC hydrogel (N). $N=4$ biological repeats. One-way ANOVA followed by Sidak's multiple comparisons test was used. Statistically significant differences ${ }^{* \star}$ adjusted $p<0.01$ and ${ }^{\star \star \star \star}$ adjusted $p<0.0001$ between conditions are shown above lines. (C) Pearson correlation between spheroid diameters and percentages of live CXCR4 ${ }^{+}$cells during 3D DE differentiation in NFC hydrogel. Statistically significant negative Pearson correlation is detected at day 2. (D) Pearson correlation between spheroid diameters and percentages of live CXCR4 ${ }^{+}$cells during 3D DE differentiation in suspension. Statistically significant negative Pearson correlation is detected at day 2.

culture systems and spheroid size influence DE differentiation of human iPSCs. Here we show that suspension culture can effectively generate DE cells from 3D human iPSC spheroids, whereas the use of biomaterials in DE differentiation may cause issues with mass transfer and spheroid formation. These findings are summarized in a schematic diagram (Figure 9). BME, a biomaterial with cell-interacting properties, produced strong cell-matrix interactions that caused 3D spheroids to spread out into monolayers (Figure 9). NFC hydrogel, a biomaterial with poor cell-interacting properties, was able to maintain $3 \mathrm{D}$ 


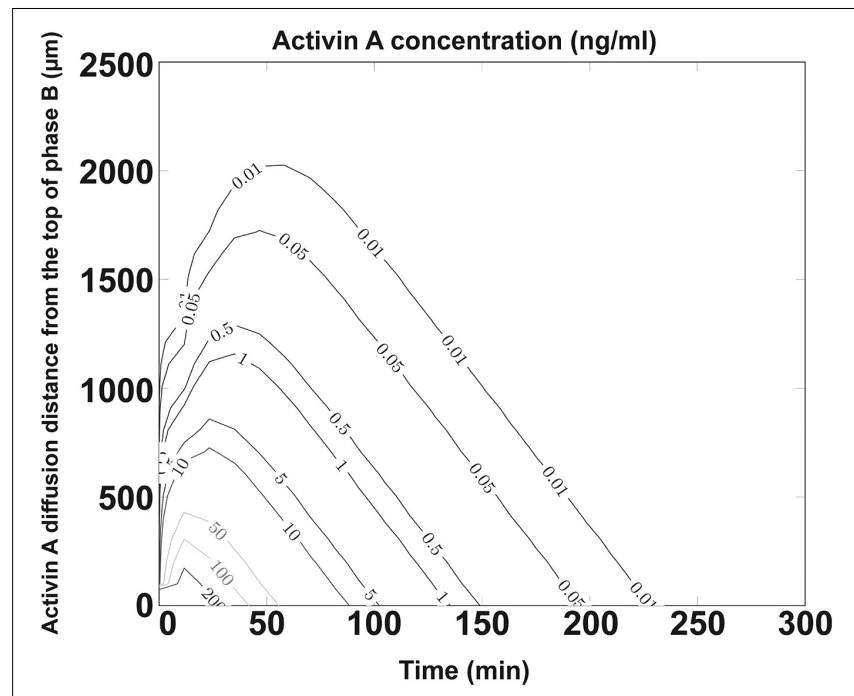

FIGURE 8 | Concentration profile of activin A protein. Diffusion coefficient set as $D_{\text {Regression }}=7.31 \times 10^{-7} \mathrm{~cm}^{2} / \mathrm{s}$ for $26.0 \mathrm{kDa}$ activin A protein and half-life $\lambda=20.3$ min (slow estimation). Activin A was diffused from the interface between phase $A$ and phase $B$ into phase $B$. Phases A and B are illustrated in Supplementary Figure 1. $Y$-axis shows the activin A diffusion distance $(\mu \mathrm{m})$ from the top of phase B. Activin A concentrations ( $\mathrm{ng} / \mathrm{ml}$ ) at different positions in phase B during 250 min after the addition of the activin A-containing differentiation medium are shown by curves. Only the top $250 \mu \mathrm{m}(8 \%)$ of the NFC hydrogel layer received $100 \mathrm{ng} / \mathrm{ml}$ activin A.

spheroid morphology during DE differentiation (Figure 9). However, NFC hydrogel impeded growth factor diffusion presumably due to its high viscosity, and therefore DE formation was less effective than in suspension culture. We also found an inverse correlation between spheroid size and the effectiveness of DE formation; smaller spheroids (with initial 200 cells per spheroid) differentiated more effectively than the larger ones (with initial 1,000 cells per spheroid). In addition, bigger spheroids (with initial 200, 500, and 1,000 cells per spheroid) formed stable cell aggregates after $24 \mathrm{~h}$, while spheroids that are too small (with initial 50 or 100 cells per spheroid) did not retain their 3D morphology in suspension or in NFC hydrogel possibly due to poor cell-cell interactions. The importance of cell-cell interaction in the formation of dermal fibroblast spheroids has recently been demonstrated by using a micropatterned hydrogel (Kim et al., 2019).

Mass transfer, also known as mass transport, is important for successful 3D cell culture and tissue engineering (Antoni et al., 2015). Sufficient mass transfer ensures the proper supply of nutrients and regulatory factors to cells and therefore generates desired cell and tissue products. Insufficient mass transfer can be caused by the presence of biomaterials and cells. Mass transfer is particularly critical when growth factors are involved because many growth factors have short half-lives, and delayed delivery to target cells results in reduced dosage to cells. By establishing a computational model to simulate growth factor diffusion, we found that activin A poorly diffused across NFC hydrogel, which may be due to the short half-life of activin A and the high viscosity of NFC hydrogel. This problem may be common for all 3D cell culture systems combining growth factors with short half-lives and highly viscose biomaterials. This issue may be solved by stabilizing growth factors or increasing the permeability of NFC hydrogel. The half-life of Wnt-3a increases when using liposomal packaging (Dhamdhere et al., 2014), so similar approaches may be applicable for activin A. However, liposomal packaging increases the radii of said growth factors, potentially hindering diffusion. Fortunately, the effect of such packaging could be predicted with the computational model described in this study. Replacing growth factors with stable small molecules is another solution. We have previously tested IDE1 as a substitute for activin $\mathrm{A}$ in DE differentiation, but unfortunately, it was ineffective (Bogacheva et al., 2018). Another study tested several small molecules in DE differentiation but still did not find an equally effective chemical to replace activin A (Tasnim et al., 2015). A high-content screening study identified two ROCK inhibitors as DE inducers in human and mouse ESCs (Korostylev et al., 2017). However, the undifferentiated ESCs used in their study were positive for DE markers, which may indicate intrinsic bias of the DE marker-positive ESC population toward endoderm differentiation as shown in an earlier study (Allison et al., 2018). Hydrogel permeability is an intrinsic property. Therefore, increasing permeability requires extensive investigation and may involve undesired changes in other hydrogel properties.

Basement membrane extract, representing another class of biomaterials that have cell-interacting properties, was also used in this study. The finding that BME could not maintain 3D spheroid morphology during DE differentiation is interesting. We speculate that this was the result of the imbalance between cell-matrix interactions and cell-cell interactions (Figure 9). It is known that undifferentiated ESCs and iPSCs can interact with BME (Xu et al., 2001). Upon embedding iPSC spheroids in BME, strong cell-BME interactions may override cell-cell interactions, thereby causing disruption of spheroid morphology. Decreasing E-cadherin expression during DE differentiation (D'Amour et al., 2005) further reduces cell-cell interactions, thereby contributing to disrupted spheroid morphology. In contrast, NFC hydrogel has negligible adhesion forces to stem cells (Harjumaki et al., 2019)minimizing cell-matrix interactions. Thus, the predominance of cell-cell interactions may account for the well maintenance of the spheroid shape in NFC hydrogel (Figure 9). These conclusions are supported by Nie et al. (2020) who previously showed with human keratinocytes that decreased cell-substrate adhesion was the main driving force in the spheroid formation and at the same time cell-cell interaction forces increased and exceeded cell-biomaterial interaction force levels.

The migrating cells in BME are presumably DE cells as shown by the immunofluorescence of SOX17 (data not shown). The reason for their death from day 4 onward could be due to the nonsupportive environment. Although DE cells can be derived from human PSCs on BME in 2D culture, DE cells, in fact, have limited ability to attach to BME as shown by the downregulation of laminin 111-specific integrins in DE cells (Kanninen et al., 2016a) and failure on re-attachment to BME after detachment (Kanninen et al., 2016b). Derivation of DE 


\section{A The balance between cell-cell interactions and cell-biomaterial interactions can affect DE spheroid morphology}

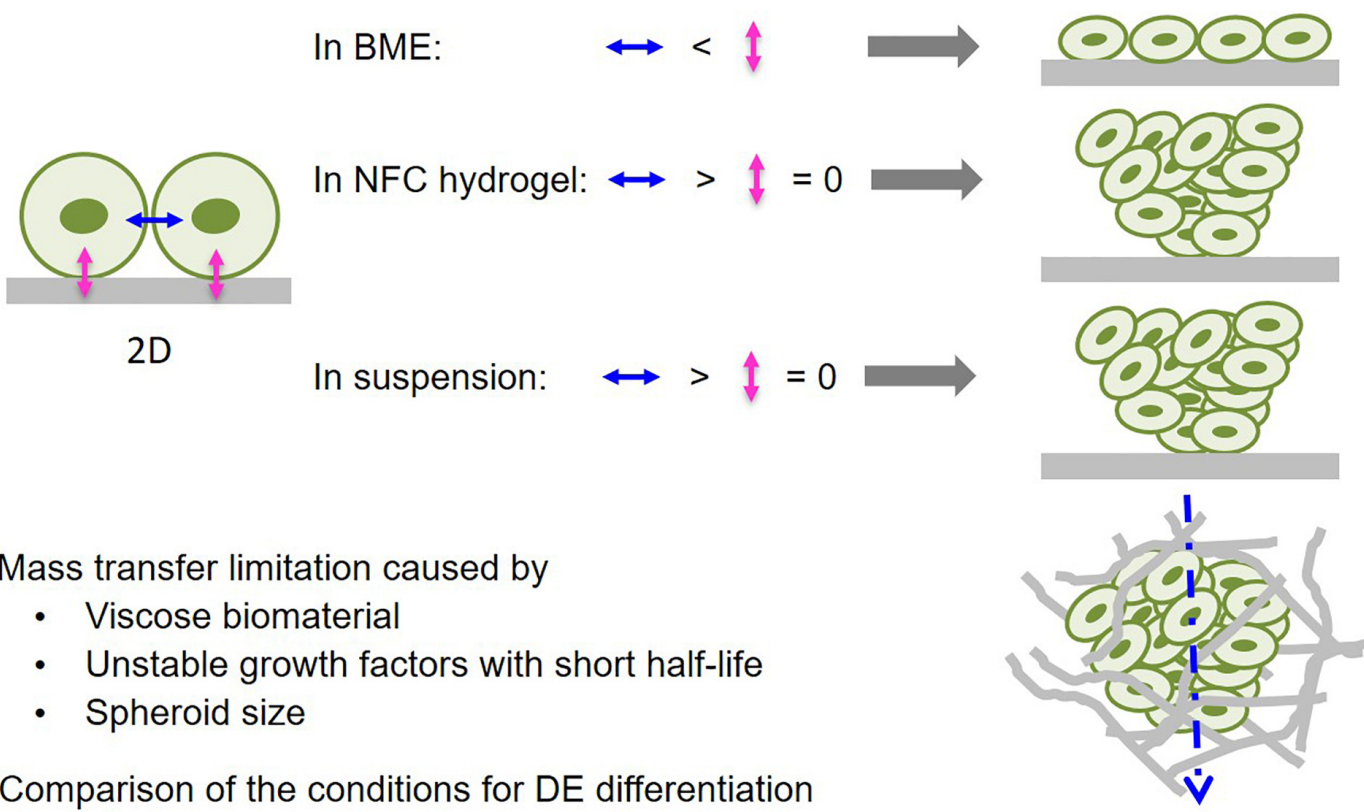

c Comparison of the conditions for DE differentiation

\begin{tabular}{|l|l|l|l|}
\hline Condition & $\begin{array}{l}\text { Dominant cell-cell } \\
\text { interactions }\end{array}$ & $\begin{array}{l}\text { Sufficient mass transfer in } \\
\text { the environment }\end{array}$ & $\begin{array}{l}\text { Optimal spheroid } \\
\text { size }\end{array}$ \\
\hline $200 \mathrm{~S}$ & + & + & + \\
\hline $1000 \mathrm{~S}$ & + & + & - \\
\hline $200 \mathrm{~N}$ & + & - & + \\
\hline $1000 \mathrm{~N}$ & + & - & - \\
\hline $200 \mathrm{BME}$ & - & Not studied & Not studied \\
\hline $1000 \mathrm{BME}$ & - & Not studied & Not studied \\
\hline
\end{tabular}

FIGURE 9 | The critical influencing parameters in 3D DE differentiation. (A) Predominance of cell-biomaterial interactions in basement membrane extract (BME) leads to disintegration of 3D spheroid morphology, while predominance of cell-cell interactions in NFC hydrogel and suspension culture retains 3D spheroid morphology. (B) Viscose biomaterial and cell mass can limit mass transfer, thereby impairing differentiation that is mediated by growth factors with short half-lives. (C) Comparison of the studied 3D culture systems in terms of their properties affecting DE differentiation effectiveness.

cells on BME in 2D culture may involve an undifferentiated PSC-produced niche, which requires further investigation.

Suspension culture is a biomaterial/scaffold-free system, meaning that there is no potential mass transfer limitation caused by biomaterials. In some cases, cells are attached to floating microcarriers. Nonetheless, all suspension cultures ensure equal supply to all spheroids. Another biomaterial/scaffold-free system that has been used in spheroid technology is the hangingdrop system. It has been used to form spheroids of dermal papilla cells in a controllable and scalable manner (Lin et al., 2016). By performing DE differentiation that involves the use of growth factor, we clearly demonstrate that suspension culture is a superior $3 \mathrm{D}$ culture system to biomaterial-based $3 \mathrm{D}$ systems because it provides equal molecular diffusion among all the spheroids, and therefore produces efficient DE differentiation. Suspension culture is scalable (Amit et al., 2010; Li et al., 2018) and can be performed in a bioreactor with tight control of cell culture conditions for mass production (Lock and Tzanakakis, 2009). Unlike biomaterial-based culture systems, suspension culture does not provide physical constraint and therefore spheroids grew faster, as shown by more significant increases in diameter in suspension culture than in NFC hydrogel culture (Supplementary Figures 4, 6).

In addition to biomaterials, cells can also limit mass transfer. Earlier studies have found that hepatocyte spheroids with diameters of more than $100 \mu \mathrm{m}$ (Glicklis et al., 2004) or human ESC and iPSC spheroids with diameters of more than $350 \mu \mathrm{m}$ (Amit et al., 2010) had cell viability rates below 
90\%. Our 4-day protocol for DE differentiation generated more than $90 \%$ viable cells (Supplementary Figure 13) in spheroids with the average diameters below $350 \mu \mathrm{m}$ (Figure 3B and Supplementary Figure 5) in all the conditions studied. Despite the high cell viability, we found that iPSC spheroids with a higher cell number had lower differentiation effectiveness. Our finding agrees with an earlier report showing that large spheroids generated fewer SOX17- and HNF3B-positive cells (Farzaneh et al., 2018). The low effectiveness of differentiation in large spheroids is presumably due to the insufficient supply of growth factors. Computational simulation of growth factor diffusion through spheroids is not straightforward because growth factor can bind to any cells on its route of diffusion. There have been computational models for oxygen permeation into a spheroid (Aleksandrova et al., 2016; Grimes and Currell, 2018), and more comprehensive models could be established to estimate protein diffusion in a cell spheroid with consideration of growth factor-receptor binding and receptor abundance. Although smaller spheroids ensure a sufficient supply of growth factor, they may not survive during DE differentiation, as shown in the current study. It is known that cadherins mediate homophilic binding between the same types of cells. DE cells express a lower level of E-cadherin and a higher level of $\mathrm{N}$-cadherin than undifferentiated ESCs and iPSCs (D'Amour et al., 2005). During DE differentiation, weaker cadherinmediated interactions could have been generated between undifferentiated PSCs and differentiating cells leading to the failure of smaller spheroids to retain their 3D morphology.

We found that cell viability inside the spheroids can be improved with the constant presence of the ROCK inhibitor Y-23720 in the differentiation media. An earlier study found a priming effect of a high concentration of Y-23720 on mesendoderm differentiation (Maldonado et al., 2016); to ensure the use of Y-23720 does not have a negative effect on DE differentiation, we analyzed its influence on the gene expression of specific pluripotency, DE, mesendoderm, and ectoderm markers. Among the three studied DE markers, only CER1 was significantly altered by 4.2 -folds by the 7 -day treatment with $10 \mu$ M Y-23720 (Supplementary Figure 2). Cer1 is a secreted protein participating in the regulation of Nodal, Wnt, and BMP signaling pathways and is a marker of the endoderm specification (Iwashita et al., 2013). However, the 4.2-fold increase is much less than the 30,000-fold increase observed during the directed DE differentiation (Figure 4E). Based on our data, the effect of Y-23720 on the DE differentiation of human PSCs was relatively mild, but its biological impact needs further investigation.

Several studies have demonstrated that $3 \mathrm{D}$ cell culture systems can produce DE cells from human ESCs and iPSCs. A small molecule IDE1 was shown to induce DE differentiation of human iPSCs in a poly( $\varepsilon$-caprolactone)-based scaffold, but the expression of HNF3B and SOX17 was only induced no more than 20-folds (Hoveizi et al., 2014). In contrast to 3D culture, IDE1 (Bogacheva et al., 2018) and its analog IDE2 (Tasnim et al., 2015) were found to be ineffective in 2D DE differentiation. Another study produced DE cells in alginate hydrogel using activin A and Wnt-3a with 800- and 300-fold induction of HNF3B and SOX17, respectively (Chayosumrit et al., 2010), whereas we observed more than 4000- and 40,000-fold increase in the expression of HNF3B and SOX17, respectively. A recent study showed that 3D DE differentiation in suspension culture has an increased proliferation coefficient and higher speed of the upregulation of DE markers in comparison with adherent culture (Yabe et al., 2019). Another study successfully produced DE aggregates in chemically defined, xeno-free suspension culture, which was demonstrated to be scalable by using bioreactors (Sahabian et al., 2019). In addition, 3D culture has been proven to improve the maturation of hepatic cells (Miki et al., 2011; Freyer et al., 2016) and pancreatic cells (Wang and Ye, 2009).

We found that the remaining problem with NFC hydrogel culture is high viscosity. Utilization of chemically stable small molecules to replace growth factors would potentially solve mass transfer limitation and thus would allow more effective use of NFC hydrogel in stem cell differentiation and spheroid formation. The fast growth kinetic in suspension culture might yield larger spheroids that can inhibit mass transfer. Dissociating spheroids followed by making smaller spheroids, for example 200 cells per spheroid, would be a strategy to maintain sufficient mass transfer during differentiation experiments.

This study demonstrates that the spheroids with initial 200 cells per spheroid in suspension culture can efficiently produce DE cells (Figure 9C). However, further work is required to quantitatively demonstrate the inverted correlation between the size of the spheroids and the expression of nuclear DE markers, which was not demonstrated in the current study due to technical limitations in flow cytometry detecting intracellular proteins. Moreover, it is necessary to assess the potential of these cells in further differentiation into endoderm derivatives, such as hepatic, pancreatic, and intestinal cells. To further differentiate DE spheroids generated in the current study, we could continue using suspension culture system to ensure sufficient mass transfer. If stable small molecules to replace growth factors are available, hydrogel-based $3 \mathrm{D}$ culture systems can also be utilized to provide physical support and constraint.

\section{CONCLUSION}

In the current study, we show how the size of the human iPSC spheroids and 3D culture conditions influence DE differentiation. We found that the ROCK inhibitor improves human iPSC spheroid viability and can be applied for the entire length of DE differentiation. The spheroid size determines the availability of growth factors as well as nutrients and oxygen supply to all the cells. Suspension culture provides sufficient mass transfer and thus generates more effective DE differentiation than NFC hydrogel-based culture. When using biomaterials, cell-matrix interaction and mass transfer should be considered because they can affect 3D cell spheroid morphology and the effectiveness of growth factor-mediated differentiation, respectively. Our findings are beneficial for the development of human iPSCderived 3D cell models, which have applications in drug research 
field for the evaluation of toxicity and efficacy of drug candidates, developmental biology studies, and regenerative medicine.

\section{DATA AVAILABILITY STATEMENT}

The original contributions presented in the study are included in the article/Supplementary Material, further inquiries can be directed to the corresponding author/s.

\section{AUTHOR CONTRIBUTIONS}

Y-RL conceived, designed, and supervised the research, carried out some of the experiments presented in the manuscript, analyzed the data, and wrote the Introduction, Results, and Discussion sections of the manuscript. MSB carried out most of the experiments presented in the manuscript, analyzed most of the data, made the figures, and wrote the Materials and Methods section and part of the Results section. RH performed preliminary DE differentiation of human PSC spheroids without spheroid size control in NFC hydrogel and suspension culture (data not shown). EF carried out some of the experiments presented in the manuscript, measured spheroid diameters, and analyzed qPCR data. AT performed preliminary DE differentiation of human PSC spheroids without spheroid size control in NFC hydrogel and suspension culture (data not shown), performed computational simulation of activin A diffusion, and wrote the modeling part of the Methods section. $\mathrm{MAB}$ helped in some experiments and measured spheroid diameters. AL provided valuable advice during research planning, analyzed qPCR data, and edited the manuscript. All authors commented on the final version of the manuscript.

\section{REFERENCES}

Adams, R., and Essex, C. (2009). Calculus: A Complete Course. North York, ON: Pearson Education Canada.

Aleksandrova, A. V., Pulkova, N. P., Gerasimenko, T. N., Anisimov, N. Y., Tonevitskaya, S. A., and Sakharov, D. A. (2016). Mathematical and experimental model of oxygen diffusion for HepaRG cell spheroids. Bull. Exp. Biol. Med. 160, 857-860. doi: 10.1007/s10517-016-3326-1

Allison, T. F., Smith, A. J. H., Anastassiadis, K., Sloane-Stanley, J., Biga, V., Stavish, D., et al. (2018). Identification and single-cell functional characterization of an endodermally biased pluripotent substate in human embryonic stem cells. Stem Cell Rep. 10, 1895-1907. doi: 10.1016/j.stemcr.2018.04.015

Amit, M., Chebath, J., Margulets, V., Laevsky, I., Miropolsky, Y., Shariki, K., et al. (2010). Suspension culture of undifferentiated human embryonic and induced pluripotent stem cells. Stem Cell Rev. 6, 248-259.

Antoni, D., Burckel, H., Josset, E., and Noel, G. (2015). Three-dimensional cell culture: a breakthrough in vivo. Int. J. Mol. Sci. 16, 5517-5527. doi: 10.3390/ ijms 16035517

Berg, H. (1993). Random Walks in Biology. Princeton, NJ: Princeton University Press.

Bergmann, S., Lawler, S. E., Qu, Y., Fadzen, C. M., Wolfe, J. M., Regan, M. S., et al. (2018). Blood-brain-barrier organoids for investigating the permeability of CNS therapeutics. Nat. Protoc. 13, 2827-2843. doi: 10.1038/s41596-018-0066-x

Bhattacharya, M., Malinen, M. M., Lauren, P., Lou, Y. R., Kuisma, S. W., Kanninen, L., et al. (2012). Nanofibrillar cellulose hydrogel promotes three-dimensional liver cell culture. J. Control. Release 164, 291-298. doi: 10.1016/j.jconrel.2012. 06.039

\section{FUNDING}

This work was supported by grants from the Academy of Finland (Nos. 294193 and 294194 to Y-RL), Finnish National Agency for Education (decision 10.10.2018/TM-18-10922 to Y-RL for MAB), and Faculty of Pharmacy, University of Helsinki to Y-RL and MY.

\section{ACKNOWLEDGMENTS}

Y-RL acknowledges Fudan University for providing research grant. MSB and Y-RL acknowledge the Doctoral Programme in Drug Research for providing MSB a Ph.D. student position at the Faculty of Pharmacy, University of Helsinki. XX acknowledges the National Natural Science Foundation of China (81473409) and Shanghai Science and Technology Innovation Fund (18140900900). We would like to thank the Light Microscopy Unit at the Institute of Biotechnology for providing confocal microscopy, Flow Cytometry Unit at the University of Helsinki for providing BD LSRFortessa Cell Analyzer, and Päivi Tammela for providing the NanoDrop spectrophotometer. The antibody MC-813-70 (SSEA-4) developed by Solter D/Knowles BB was obtained from the Developmental Studies Hybridoma Bank developed under the auspices of the NICHD and maintained by The University of Iowa, Department of Biology, Iowa City, IA, United States.

\section{SUPPLEMENTARY MATERIAL}

The Supplementary Material for this article can be found online at: https://www.frontiersin.org/articles/10.3389/fcell.2021. 726499/full\#supplementary-material

Bogacheva, M. S., Khan, S., Kanninen, L. K., Yliperttula, M., Leung, A. W., and Lou, Y. R. (2018). Differences in definitive endoderm induction approaches using growth factors and small molecules. J. Cell. Physiol. 233, 3578-3589. doi: $10.1002 /$ jcp. 26214

Broguiere, N., Isenmann, L., Hirt, C., Ringel, T., Placzek, S., Cavalli, E., et al. (2018). Growth of epithelial organoids in a defined hydrogel. Adv. Mater. 30:e1801621.

Candiello, J., Grandhi, T. S. P., Goh, S. K., Vaidya, V., Lemmon-Kishi, M., Eliato, K. R., et al. (2018). 3D heterogeneous islet organoid generation from human embryonic stem cells using a novel engineered hydrogel platform. Biomaterials 177, 27-39. doi: 10.1016/j.biomaterials.2018.05.031

Chayosumrit, M., Tuch, B., and Sidhu, K. (2010). Alginate microcapsule for propagation and directed differentiation of hESCs to definitive endoderm. Biomaterials 31, 505-514. doi: 10.1016/j.biomaterials.2009.09.071

Chen, G., Hou, Z., Gulbranson, D. R., and Thomson, J. A. (2010). Actin-myosin contractility is responsible for the reduced viability of dissociated human embryonic stem cells. Cell Stem Cell 7, 240-248. doi: 10.1016/j.stem.2010. 06.017

Chua, C. W., Shibata, M., Lei, M., Toivanen, R., Barlow, L. J., Bergren, S. K., et al. (2014). Single luminal epithelial progenitors can generate prostate organoids in culture. Nat. Cell Biol. 16, 951-954. doi: 10.1038/ncb3047

D’Amour, K. A., Agulnick, A. D., Eliazer, S., Kelly, O. G., Kroon, E., and Baetge, E. E. (2005). Efficient differentiation of human embryonic stem cells to definitive endoderm. Nat. Biotechnol. 23, 1534-1541. doi: 10.1038/nbt1163

Dhamdhere, G. R., Fang, M. Y., Jiang, J., Lee, K., Cheng, D., Olveda, R. C., et al. (2014). Drugging a stem cell compartment using Wnt3a protein as a therapeutic. PLoS One 9:e83650. doi: 10.1371/journal.pone.008 3650 doi: 10.1371 /journal.pone.0083650 
Dye, B. R., Hill, D. R., Ferguson, M. A., Tsai, Y. H., Nagy, M. S., Dyal, R., et al. (2015). In vitro generation of human pluripotent stem cell derived lung organoids. Elife 4:e05098.

Farzaneh, Z., Najarasl, M., Abbasalizadeh, S., Vosough, M., and Baharvand, H. (2018). Developing a cost-effective and scalable production of human hepatic competent endoderm from size-controlled pluripotent stem cell aggregates. Stem Cells Dev. 27, 262-274. doi: 10.1089/scd.2017.0074

Fatehullah, A., Tan, S. H., and Barker, N. (2016). Organoids as an in vitro model of human development and disease. Nat. Cell Biol. 18, 246-254. doi: 10.1038/ ncb3312

Freyer, N., Knospel, F., Strahl, N., Amini, L., Schrade, P., Bachmann, S., et al. (2016). Hepatic differentiation of human induced pluripotent stem cells in a perfused three-dimensional multicompartment bioreactor. Biores. Open Access 5, 235-248. doi: 10.1089/biores.2016.0027

Gjorevski, N., Sachs, N., Manfrin, A., Giger, S., Bragina, M. E., Ordonez-Moran, P., et al. (2016). Designer matrices for intestinal stem cell and organoid culture. Nature 539, 560-564. doi: 10.1038/nature20168

Glicklis, R., Merchuk, J. C., and Cohen, S. (2004). Modeling mass transfer in hepatocyte spheroids via cell viability, spheroid size, and hepatocellular functions. Biotechnol. Bioeng. 86, 672-680. doi: 10.1002/bit.20086

Grimes, D. R., and Currell, F. J. (2018). Oxygen diffusion in ellipsoidal tumour spheroids. J. R. Soc. Interface 15:20180256. doi: 10.1098/rsif.2018. 0256

Hanawa, M., Takayama, K., Sakurai, F., Tachibana, M., and Mizuguchi, H. (2017). Hepatocyte nuclear factor 4 alpha promotes definitive endoderm differentiation from human induced pluripotent stem cells. Stem Cell Rev. Rep. 13, 542-551. doi: 10.1007/s12015-016-9709-x

Harjumaki, R., Nugroho, R. W. N., Zhang, X., Lou, Y. R., Yliperttula, M., ValleDelgado, J. J., et al. (2019). Quantified forces between HepG2 hepatocarcinoma and WA07 pluripotent stem cells with natural biomaterials correlate with in vitro cell behavior. Sci. Rep. 9:7354.

Hay, D. C., Fletcher, J., Payne, C., Terrace, J. D., Gallagher, R. C., Snoeys, J., et al. (2008). Highly efficient differentiation of hESCs to functional hepatic endoderm requires ActivinA and Wnt3a signaling. Proc. Natl. Acad. Sci. U.S.A. 105, 12301-12306. doi: 10.1073/pnas.0806522105

Hohwieler, M., Illing, A., Hermann, P. C., Mayer, T., Stockmann, M., Perkhofer, L., et al. (2017). Human pluripotent stem cell-derived acinar/ductal organoids generate human pancreas upon orthotopic transplantation and allow disease modelling. Gut 66, 473-486. doi: 10.1136/gutjnl-2016-312423

Hoveizi, E., Khodadadi, S., Tavakol, S., Karima, O., and Nasiri-Khalili, M. A. (2014). Small molecules differentiate definitive endoderm from human induced pluripotent stem cells on PCL scaffold. Appl. Biochem. Biotechnol. 173, 17271736. doi: 10.1007/s12010-014-0960-9

Iwashita, H., Shiraki, N., Sakano, D., Ikegami, T., Shiga, M., Kume, K., et al. (2013). Secreted cerberus 1 as a marker for quantification of definitive endoderm differentiation of the pluripotent stem cells. PLoS One 8:e64291. doi: 10.1371/ journal.pone.0064291

Johnson, K. E., Makanji, Y., Temple-Smith, P., Kelly, E. K., Barton, P. A., Al-Musawi, S. L., et al. (2016). Biological activity and in vivo half-life of pro-activin A in male rats. Mol. Cell. Endocrinol. 422, 84-92. doi: 10.1016/j.mce.2015.12.007

Kanninen, L. K., Harjumaki, R., Peltoniemi, P., Bogacheva, M. S., Salmi, T., Porola, P., et al. (2016a). Laminin-511 and laminin-521-based matrices for efficient hepatic specification of human pluripotent stem cells. Biomaterials 103, 86-100. doi: 10.1016/j.biomaterials.2016.06.054

Kanninen, L. K., Porola, P., Niklander, J., Malinen, M. M., Corlu, A., GuguenGuillouzo, C., et al. (2016b). Hepatic differentiation of human pluripotent stem cells on human liver progenitor HepaRG-derived acellular matrix. Exp. Cell Res. 341, 207-217. doi: 10.1016/j.yexcr.2016.02.006

Kim, E. M., Lee, Y. B., Byun, H., Chang, H. K., Park, J., and Shin, H. (2019). Fabrication of spheroids with uniform size by self-assembly of a micro-scaled cell sheet (muCS): the effect of cell contraction on spheroid formation. ACS Appl. Mater. Interfaces 11, 2802-2813. doi: 10.1021/acsami.8b 18048

Kim, Y., Kim, H., Ko, U. H., Oh, Y., Lim, A., Sohn, J. W., et al. (2016). Islet-like organoids derived from human pluripotent stem cells efficiently function in the glucose responsiveness in vitro and in vivo. Sci. Rep. $6: 35145$.
Korostylev, A., Mahaddalkar, P. U., Keminer, O., Hadian, K., Schorpp, K., Gribbon, P., et al. (2017). A high-content small molecule screen identifies novel inducers of definitive endoderm. Mol. Metab. 6, 640-650. doi: 10.1016/j.molmet.2017. 04.009

Kumar, S. V., Er, P. X., Lawlor, K. T., Motazedian, A., Scurr, M., Ghobrial, I., et al. (2019). Kidney micro-organoids in suspension culture as a scalable source of human pluripotent stem cell-derived kidney cells. Development 146:dev172361.

Li, X., Ma, R., Gu, Q., Liang, L., Wang, L., Zhang, Y., et al. (2018). A fully defined static suspension culture system for large-scale human embryonic stem cell production. Cell Death Dis. 9:892.

Lin, B., Miao, Y., Wang, J., Fan, Z., Du, L., Su, Y., et al. (2016). Surface tension guided hanging-drop: producing controllable $3 \mathrm{D}$ spheroid of high-passaged human dermal papilla cells and forming inductive microtissues for hair-follicle regeneration. ACS Appl. Mater. Interfaces 8, 5906-5916. doi: 10.1021/acsami. 6b00202

Lock, L. T., and Tzanakakis, E. S. (2009). Expansion and differentiation of human embryonic stem cells to endoderm progeny in a microcarrier stirred-suspension culture. Tissue Eng. Part A 15, 2051-2063. doi: 10.1089/ten.tea.2008.0455

Lou, Y. R., Kanninen, L., Kuisma, T., Niklander, J., Noon, L. A., Burks, D., et al. (2014). The use of nanofibrillar cellulose hydrogel as a flexible threedimensional model to culture human pluripotent stem cells. Stem Cells Dev. 23, 380-392. doi: 10.1089/scd.2013.0314

Lou, Y. R., and Leung, A. W. (2018). Next generation organoids for biomedical research and applications. Biotechnol. Adv. 36, 132-149. doi: 10.1016/j. biotechadv.2017.10.005

Maldonado, M., Luu, R. J., Ramos, M. E., and Nam, J. (2016). ROCK inhibitor primes human induced pluripotent stem cells to selectively differentiate towards mesendodermal lineage via epithelial-mesenchymal transition-like modulation. Stem Cell Res. 17, 222-227. doi: 10.1016/j.scr.2016.07.009

Malinen, M. M., Kanninen, L. K., Corlu, A., Isoniemi, H. M., Lou, Y. R., Yliperttula, M. L., et al. (2014). Differentiation of liver progenitor cell line to functional organotypic cultures in 3D nanofibrillar cellulose and hyaluronan-gelatin hydrogels. Biomaterials 35, 5110-5121. doi: 10.1016/j.biomaterials.2014.03.020

McLean, A. B., D’Amour, K. A., Jones, K. L., Krishnamoorthy, M., Kulik, M. J., Reynolds, D. M., et al. (2007). Activin a efficiently specifies definitive endoderm from human embryonic stem cells only when phosphatidylinositol 3-kinase signaling is suppressed. Stem Cells 25, 29-38. doi: 10.1634/stemcells.2006-0219

Miki, T., Ring, A., and Gerlach, J. (2011). Hepatic differentiation of human embryonic stem cells is promoted by three-dimensional dynamic perfusion culture conditions. Tissue Eng. Part C Methods 17, 557-568. doi: 10.1089/ten. tec. 2010.0437

Nelson, P. (2013). Biological Physics. New York, NY: W. H. Freeman.

Nie, Y., Xu, X., Wang, W., Ma, N., and Lendlein, A. (2020). Spheroid formation of human keratinocyte: balancing between cell-substrate and cell-cell interaction. Clin. Hemorheol. Microcirc. 76, 329-340. doi: 10.3233/ch-209217

Nowak, M., Freudenberg, U., Tsurkan, M. V., Werner, C., and Levental, K. R. (2017). Modular GAG-matrices to promote mammary epithelial morphogenesis in vitro. Biomaterials 112, 20-30. doi: 10.1016/j.biomaterials. 2016.10.007

Pfaffl, M. W. (2001). A new mathematical model for relative quantification in real-time RT-PCR. Nucleic Acids Res. 29:e45.

Rahman, M., and Sadygov, R. G. (2017). Predicting the protein half-life in tissue from its cellular properties. PLoS One 12:e0180428. doi: 10.1371/journal.pone. 0180428

Sahabian, A., Sgodda, M., Naujok, O., Dettmer, R., Dahlmann, J., Manstein, F., et al. (2019). Chemically-defined, xeno-free, scalable production of hPSC-derived definitive endoderm aggregates with multi-lineage differentiation potential. Cells 8:1571. doi: 10.3390/cells8121571

Schuldiner, M., Yanuka, O., Itskovitz-Eldor, J., Melton, D. A., and Benvenisty, N. (2000). Effects of eight growth factors on the differentiation of cells derived from human embryonic stem cells. Proc. Natl. Acad. Sci. U.S.A. 97, 11307-11312. doi: 10.1073/pnas.97.21.11307

Spence, J. R., Mayhew, C. N., Rankin, S. A., Kuhar, M. F., Vallance, J. E., Tolle, K., et al. (2011). Directed differentiation of human pluripotent stem cells into intestinal tissue in vitro. Nature 470, 105-109.

Tasnim, F., Phan, D., Toh, Y. C., and Yu, H. (2015). Cost-effective differentiation of hepatocyte-like cells from human pluripotent stem cells using small molecules. Biomaterials 70, 115-125. doi: 10.1016/j.biomaterials.2015.08.002 
Teo, A. K., Ali, Y., Wong, K. Y., Chipperfield, H., Sadasivam, A., Poobalan, Y., et al. (2012). Activin and BMP4 synergistically promote formation of definitive endoderm in human embryonic stem cells. Stem Cells 30, 631-642. doi: 10. 1002/stem.1022

Teo, A. K., Arnold, S. J., Trotter, M. W., Brown, S., Ang, L. T., Chng, Z., et al. (2011). Pluripotency factors regulate definitive endoderm specification through eomesodermin. Genes Dev. 25, 238-250. doi: 10.1101/gad.607311

Wang, H., Luo, X., Yao, L., Lehman, D. M., and Wang, P. (2015). Improvement of cell survival during human pluripotent stem cell definitive endoderm differentiation. Stem Cells Dev. 24, 2536-2546. doi: 10.1089/scd.2015.0018

Wang, X., and Ye, K. (2009). Three-dimensional differentiation of embryonic stem cells into islet-like insulin-producing clusters. Tissue Eng. Part A 15, 1941-1952. doi: 10.1089/ten.tea.2008.0181

Wang, Z., Oron, E., Nelson, B., Razis, S., and Ivanova, N. (2012). Distinct lineage specification roles for NANOG, OCT4, and SOX2 in human embryonic stem cells. Cell Stem Cell 10, 440-454. doi: 10.1016/j.stem.2012.02.016

Watanabe, K., Ueno, M., Kamiya, D., Nishiyama, A., Matsumura, M., Wataya, T., et al. (2007). A ROCK inhibitor permits survival of dissociated human embryonic stem cells. Nat. Biotechnol. 25, 681-686. doi: 10.1038/nbt1310

Wimmer, R. A., Leopoldi, A., Aichinger, M., Wick, N., Hantusch, B., Novatchkova, M., et al. (2019). Human blood vessel organoids as a model of diabetic vasculopathy. Nature 565, 505-510. doi: 10.1038/s41586-018-0858-8

Xu, C., Inokuma, M. S., Denham, J., Golds, K., Kundu, P., Gold, J. D., et al. (2001). Feeder-free growth of undifferentiated human embryonic stem cells. Nat. Biotechnol. 19, 971-974. doi: 10.1038/nbt1001-971

Yabe, S. G., Nishida, J., Fukuda, S., Takeda, F., Nashiro, K., Ibuki, M., et al. (2019). Definitive endoderm differentiation is promoted in suspension cultured human
iPS-derived spheroids more than in adherent cells. Int. J. Dev. Biol. 63, 271-280. doi: $10.1387 / \mathrm{ijdb} .180251$ sy

Yu, J., Vodyanik, M. A., Smuga-Otto, K., Antosiewicz-Bourget, J., Frane, J. L., Tian, S., et al. (2007). Induced pluripotent stem cell lines derived from human somatic cells. Science 318, 1917-1920.

Zorn, A. M., and Wells, J. M. (2009). Vertebrate endoderm development and organ formation. Annu. Rev. Cell Dev. Biol. 25, 221-251. doi: 10.1146/annurev.cellbio. 042308.113344

Conflict of Interest: The authors declare that the research was conducted in the absence of any commercial or financial relationships that could be construed as a potential conflict of interest.

Publisher's Note: All claims expressed in this article are solely those of the authors and do not necessarily represent those of their affiliated organizations, or those of the publisher, the editors and the reviewers. Any product that may be evaluated in this article, or claim that may be made by its manufacturer, is not guaranteed or endorsed by the publisher.

Copyright (C 2021 Bogacheva, Harjumäki, Flander, Taalas, Bystriakova, Yliperttula, Xiang, Leung and Lou. This is an open-access article distributed under the terms of the Creative Commons Attribution License (CC BY). The use, distribution or reproduction in other forums is permitted, provided the original author(s) and the copyright owner(s) are credited and that the original publication in this journal is cited, in accordance with accepted academic practice. No use, distribution or reproduction is permitted which does not comply with these terms. 